\title{
Maternal Obesity Dysregulates Fetal Hematopoietic Stem and Progenitor Cell Development in Rhesus Macaques
}

Suhas Sureshchandra ${ }^{1}$, Jacob J. Robino ${ }^{2}$, Devorah Goldman ${ }^{3}$, Diana Takahashi ${ }^{2}$, Weining Shen $^{4}$ Jon D. Hennebold ${ }^{5,6}$, Charles T. Roberts Jr. ${ }^{2,5}$, Benjamin J. Burwitz ${ }^{7,8}$, Ilhem Messaoudi ${ }^{1}$ and Oleg Varlamov ${ }^{2 *}$

${ }^{1}$ Department of Molecular Biology and Biochemistry, School of Biological Sciences; Institute for Immunology; Center for Virus Research, ${ }^{4}$ Department of Statistics, University of California-Irvine, Irvine, CA 92697, USA; Divisions of ${ }^{2}$ Cardiometabolic Health, ${ }^{5}$ Reproductive and Developmental Sciences, ${ }^{7}$ Pathobiology and Immunology, Oregon National Primate Research Center; ${ }^{8}$ Vaccine \& Gene Therapy Institute, Beaverton, OR, 97006, USA; Departments of ${ }^{3}$ Pediatrics and ${ }^{6}$ Obstetrics and Gynecology, Oregon Health \& Science University, Portland, OR 97239, USA

*Corresponding author: Oleg Varlamov, MD, PhD, Oregon National Primate Research Center, 505 NW 185 ${ }^{\text {th }}$ Ave, Beaverton OR 97006, USA varlamov@ohsu.edu; 503-346-5377

Running title: Maternal obesity dysregulates fetal hematopoiesis

Key words: adipocytes; B-lymphocytes; bone marrow; fetal development; hematopoietic stem and progenitor cells; high-fat diet, macrophages, monocytes, nonhuman primates; maternal programming; myelopoiesis; obesity; single-cell RNA-sequencing; western-style diet 


\begin{abstract}
Infants from obese moms have an increased susceptibility to immune dysregulation. However, the mechanisms by which maternal obesity alters fetal hematopoiesis remain largely unknown. Here, we determined the impact of maternal consumption of an obesogenic western-style diet (WSD) on hematopoietic development in fetal rhesus macaques using a combination of phenotypic, functional, and genomic assays. We demonstrate that maternal WSD resulted in accelerated fetal growth and altered fetal hematopoiesis. Specifically, single-cell RNA sequencing analysis of fetal bone marrow HSPCs showed that maternal WSD altered the transcriptional program of the common lymphoid progenitors and decreased the frequencies of bone marrow Bcells and NK-cells. Despite an expansion of monocyte progenitors in FBM, fetal blood monocytes from the WSD group demonstrated a blunted response to bacterial lipopolysaccharide. Furthermore, maternal WSD led to poor engraftment of fetal HSPCs in nonlethally irradiated immunodeficient NOD/SCID/IL2 $r \gamma^{-/-}$mice. Collectively, this study demonstrates that maternal WSD dysregulates fetal HSPC development.
\end{abstract}




\section{INTRODUCTION}

Accumulating evidence suggests that maternal obesity provides maladaptive intrauterine cues to the developing offspring, predisposing the offspring to infection and development of chronic disease (Rastogi et al., 2015; Suk et al., 2016). Recent work in humans (Sureshchandra et al., 2017; Wilson et al., 2015; Wilson and Messaoudi, 2015) and animal models (Friedman, 2018) has demonstrated that pregravid obesity is associated with immune dysregulation in the offspring. While our understanding of the transgenerational impact of maternal obesity on the offspring mature immune system is beginning to emerge, the mechanisms that underlie this modulation of the fetal hematopoietic system during development are still unclear.

During development, hematopoiesis occurs in several waves that involve the migration of hematopoietic stem and progenitor cells (HSPCs) between different hematopoietic organs (Gao et al., 2018). Following the emergence of HSPCs in the aorta gonad mesonephros during the first trimester, the fetal liver becomes the main hematopoietic niche for HSPC expansion (Supp. Figure 1A) (Bowie et al., 2007; Morrison et al., 1995). Epigenetic regulation of HSPCs is established during embryonic development and contributes to lineage-specific transcriptional responses and hematopoietic lineage output (Cedar and Bergman, 2011). During the third trimester, HSPCs migrate from the fetal liver to the fetal bone marrow (FBM), representing an essential developmental stage associated with a transition to the postnatal hematopoiesis (Gao et al., 2018). Therefore, alterations in the maternal environment may exert long-lasting effects on offspring immunity by reprogramming cellular output and lineage commitment in the FBM and other hematopoietic sites (Friedman, 2018).

Studies using day 14.5 mouse fetal livers (comparable to human gestation week 8 ) have demonstrated that maternal high-fat diet (HFD) and obesity compromise HSPC self-renewal and biases differentiation towards the myeloid lineage (Kamimae-Lanning et al., 2015). Given that fetal HSPCs are transcriptionally dynamic cells and that their function is highly dependent on niche-specific fetal microenvironment signals, we hypothesized that maternal obesity may 
influence fetal hematopoiesis through both intrinsic (e.g., programming of fetal HSPCs) and extrinsic (e.g., change in the development of FBM niches required for HSPC homing and differentiation) mechanisms. Here, we tested this hypothesis by leveraging the rhesus macaque model, whose hematopoietic system is highly similar to that of humans (Kim et al., 2014; Radtke et al., 2019; Sykes and Scadden, 2013; Wu et al., 2018). Obesity was induced in dams using a Western-style diet (WSD) and FBM samples obtained from gestational day (GD) 130 rhesus macaques (Bishop et al., 2018; True et al., 2017) were examined.

Using a combination of flow cytometry and single-cell RNA-sequencing (scRNA-Seq), we demonstrate that maternal WSD altered the development of fetal hematopoietic progenitors, resulting in a decrease in the frequencies of FBM lymphocytes and impaired functional responses of peripheral blood monocytes. Finally, rhesus CD34+ HSPCs isolated from fetuses of WSD dams transplanted into immunodeficient NOD/SCID/IL2r $\gamma-/-$ (NSG) mice exhibited poor engraftment in both the BM and the spleen and the accumulation of myeloid cells in the spleen. These findings collectively suggest that an obesogenic maternal diet dysregulates HSPC development. 


\section{RESULTS}

Maternal weight during pregnancy is positively associated with fetal weight.

To address the impact of maternal obesity on FBM development and hematopoiesis, we studied two experimental groups of female rhesus macaques, including those that consumed either a low-fat control diet or those consuming a calorie-rich high-fat WSD for 3 years starting at 2.5 years of age (near menarche) (Figure 1A). Animals consuming the WSD were consistently heavier and exhibited significantly greater percentage total body fat than control animals, as previously reported (Carbone et al., 2019; Varlamov et al., 2017) (Figures 1B and C). At 5.5 years of age, animals underwent timed-mated breeding (Bishop et al., 2021). WSD-fed animals remained heavier than control dams during pregnancy (Supplementary Table S1). GD130-135 fetuses (last trimester, Figure 1A and Supplementary Figure 1A) were obtained from the WSD and control dams by a cesarean section (C-section). Maternal and umbilical artery glucose levels were elevated in the WSD group (Supplementary Tables S1 and S2). There were no statistically significant group differences in fetal weight, crown-rump length, and other fetal anthropometric parameters (Figures 1D and E and Supplementary Table S2). However, maternal weight at Csection was significantly correlated with fetal weight and crown-rump length (Figure 1F and Supplementary Table S3). Furthermore, maternal weight at C-section showed a positive correlation with fetal retroperitoneal white adipose tissue and spleen weights (Supplementary Table S3). Additionally, maternal fasting insulin levels before pregnancy correlated with fetal crown-rump length (Supplementary Table S3).

To elucidate whether a maternal WSD impacts FBM development, we performed histological analyses of fetal femurs (Figure 1G). The medullary BM cavities of fetal femurs derived from the WSD group were already developed, while the control femurs exhibited trabecular morphology and the lack of the central BM cavity (Figure 1G, arrows). Additionally, this analysis revealed that the femurs of WSD fetuses were densely populated by BM adipocytes, while the femurs of control fetuses contained fewer BM adipocytes (Figure 1G, H). The red 
marrow of the control femurs was sub-compartmentalized into small pockets formed by ossified trabeculae. In contrast, the red marrow of WSD femurs was localized to the BM cavity in close proximity to BM adipocytes (Figure 1G, arrow), which resembles the structural organization of the femurs in juvenile and adult rhesus macaques (Robino et al., 2020). Collectively, our anthropometric and histological analyses demonstrate that maternal weight during pregnancy positively correlates with fetal growth and induces premature FBM adipogenesis and a postnatal pattern of bone development (Supplementary Figure 1A).

\section{Fetal macaque HSPCs segregate into discrete progenitor populations.}

We next asked if maternal WSD alters the HSPC subpopulations and/or immune cell phenotype of the FBM. Mononuclear cells were isolated from GD130 fetal tibias and frequencies of CD34+ HSPCs were measured using flow cytometry (Figure 1A). Overall frequency of CD34+ cells within the FBM mononuclear cell population was comparable between the two groups (Figure 1I). To determine the impact of maternal WSD on BM hematopoietic progenitor subsets, HSPCs were enriched using rhesus-specific anti-CD34 antibodies and magnetic beads before being profiled using droplet-based scRNA-Seq (Figure 1A). Dimension reduction and clustering of CD34+ single-cell RNA profiles ( $n=3 /$ group) revealed 21 cell clusters (Supplementary Figures 1B and 1C, Supplementary Table S4). Consolidation of redundant clusters resulted in 11 major hematopoietic progenitor clusters (Figure 2A and Supplementary Figure 1B). These clusters were annotated using both supervised and semi-supervised approaches based on markers highly expressed in each cluster relative to the remaining clusters (Supplementary Table S4). This approach allowed us to identify the cluster of hematopoietic stem cells (HSCs) expressing high levels of CD34 and HOPX, proliferating HSCs expressing MKI67, megakaryocytic-erythroid progenitors (MEPs) expressing KLF1 and HBA, common lymphoid progenitors (CLPs) expressing IL7R and DNTT, dendritic cell (DC) precursors (pre-conventional DCs, or cDCs expressing CCR2, MAMU-DRA and CD74; pre-plasmacytoid DCs, or pDCs expressing IRF7 and CCL3, granulocyte- 
myeloid progenitors (GMPs) expressing ELANE and MPO; common monocyte progenitors (cMoPs) expressing OAZ1 and $L Y Z$, and pro-monocytes expressing S100A8, IL1B and VCAN (Figures 2A and 2B). These data establish, for the first time, the single-cell transcriptional landscape of fetal nonhuman primate (NHP) CD34+ HSPCs.

\section{Maternal WSD alters B-cell development in FBM.}

Maternal WSD resulted in the dramatic shifts in the composition of FBM HSPC clusters (Supplementary Figure 1C and Figure 2C), notably a reduction in MEPs (Figure 2C, region 4) that was accompanied by an expansion of cMoPs/GMPs, pro-monocytes (Figure 2C, region 2), and CLPs (Figure 2C, region 1). The CLP cluster was further sub-divided into CLP-1 (expressing the T-cell marker CD3D), CLP-2 (expressing the B-cell markers CD79B and MAMU-DRA), and proliferating CLPs (expressing high levels of MKI67) (Supplementary Figures 2A and 2B). This subclassification revealed that the primary contributors to CLP cluster expansion with maternal WSD were CLP-2 and proliferating CLPs (Figure 2D). Pathway analysis of differentially expressed genes within CLPs revealed the up-regulation of MAPK signaling, toll-like receptor (TLR), and tumor necrosis factor- $\alpha,(T N F \alpha)$ pathways, but down-regulation of mRNAs involved in oxidative phosphorylation and the tricarboxylic acid (TCA) cycle, mRNA transport, cell cycle, and leukocyte migration pathways (Figure 2E and Supplementary Table S5).

Despite the increased proportion of B-cell precursors (CLP-2) (Figure 2D), module scoring revealed a significant down-regulation of $B$-cell receptor (BCR) signaling genes with WSD (Figure 2F and Supplementary Table S5). Moreover, the analysis of CD34-CD45+ immune cells in the FBM using flow cytometry showed reduced levels of B-lymphocytes (Supplementary Figure 2C and Figure 2G). In contrast, T-cell receptor (TCR) signaling was up-regulated with WSD in CLPs, primarily within the CLP-1 cluster (Figure 2H). However, the levels of CD3+ T-lymphocytes in the FBM were not significantly different between the two groups (Figure 2I). Finally, the frequency of natural killer (NK)-cells was reduced in the FBM of fetuses from WSD-fed dams (Figure 2J). In 
summary, maternal WSD induced the proliferation of FBM lymphoid progenitors at the expense of their maturation, resulting in a reduction in the frequencies of B-lymphocytes and NK-cells in the FBM.

\section{Maternal WSD alters myeloid cell development in FBM.}

Trajectory analysis of the myeloid branch confirmed the developmental transition of fetal HSPCs from less mature GMPs to mature pro-monocytes via cMoP (Figures 2C, 3A and Supplementary Figure 3A). Maternal exposure to WSD was associated with a reduction in GMPs but an enrichment of mature cMoPs and pro-monocytes in fetal HSPCs (Figure 3B). A direct comparison of cellular states within the myeloid trajectory showed an accelerated development of myeloid cell progenitors (HSCs, GMPs) with maternal WSD exposure (Figure 3C). In line with this accelerated maturation, differential analysis of the HSCs (Supplementary Table S5) revealed up-regulation of pathways associated with myeloid cell maturity, including NOD-like receptor (NLR), TLR, and TNF $\alpha$ signaling pathways (Figure 3D). Moreover, single-cell profiles of HSCs suggested that maternal WSD exposure reduced expression of genes involved in regulating RNA transport, BCR signaling, and cell adhesion (Figure 3D).

Despite the accelerated maturation within the myeloid lineage, multiple cytokine and chemokine signaling responses were significantly reduced in myeloid cell precursors from fetuses exposed to WSD vs chow, suggesting functional dysregulation in fetal myeloid cells (Figure 3E). We next asked if this cellular rewiring of myeloid cell precursors with WSD impacts the phenotypic composition of mature myeloid cells in the FBM. We used cell granularity, and surface expression of HLA-DR, CD14, and CD163, to distinguish FBM monocytes (less granular CD14+HLADR++CD163-) and FBM stromal macrophages (more granular CD14+HLA-DRlo/-CD163+) using flow cytometry (Supplementary Figure 3B). Although, no significant differences in the frequencies of FBM monocytes and stromal macrophages were observed (Supplementary Figure 4C) with maternal WSD consumption, the surface expression of TLR4 and CD86 were 
elevated in stromal macrophages but not in FBM monocytes (Figure 3F). Moreover, FBM stromal macrophages from the WSD group produced significantly higher levels of TNF $\alpha$ than those from the control group following stimulation with the TLR1/2 ligand Pam3CSK4 (Figure 3G and Supplementary Figure 3D). Additionally, the magnitude of the TNF $\alpha$ response to Pam3CSK4 by fetal stromal macrophages positively correlated with maternal body fat measured prior to pregnancy (Figure $\mathbf{3 H}$ ). In contrast, FBM monocytes did not respond to stimulation with bacterial synthetic ligands (Supplementary Figure 3E) and exhibited significantly reduced surface expression of HLA-DR (Figure 3F). These results demonstrate maternal WSD/obesity accelerates the maturation of the monocytic lineage in the FBM but had no significant effect on the frequencies of monocytes and stromal macrophages. Nevertheless, maternal WSD/obesity was associated with a heightened activation and inflammatory response by FBM stromal macrophages.

\section{Maternal WSD impairs the regenerative properties of FBM HSPCs in vivo.}

We next asked if maternal WSD consumption/obesity altered the functional properties of fetal HSPCs (Figure 1A). We transplanted FBM CD34+ HSPCs into nonlethally irradiated immunodeficient NOD/SCID/IL2r $\gamma$-/- (NSG) mice that were maintained on low-fat chow throughout the engraftment period (Figure 4A). Rhesus macaque chimerism levels in the blood of NSG mice was determined 13 weeks later, using NHP- and mouse-specific anti-CD45 antibodies (Supplementary Figure 4A). Mice injected with fetal CD34+ cells isolated from the WSD group exhibited higher levels of blood chimerism compared to mice injected with control fetal CD34+ cells (Figure 4B). On the other hand, levels of CD34+ HSPCs in the spleen and the BM were lower in the mice engrafted with CD34+ HSPCs from the WSD group compared to those engrafted with cells from the control group (Figure 4C and Supplementary Figures 4C and 4G). In the BM of NSG mice, we observed no group differences in frequencies of mature lymphoid cells 
(Supplementary Figures 4C and 4D). However, frequencies of mature myeloid cells (Figure 4D and Supplementary Figure 4E), including Mac387+ macrophages in the BM, were significantly decreased with a maternal WSD (Figure 4D and Supplementary Figure 4F).

In contrast to BM, the spleens (Supplementary Figure 4G) of NSG mice engrafted with fetal HSPCs from WSD dams harbored elevated frequencies of T-cells (Figure 4E) and reduced NK-cell frequencies $(p<0.05)$ (Figure 4E), but a significant increase in CD11b+Mac387+ macrophages $(p<0.0001)$ (Figure 4F and Supplementary Figure 4F). Similarly, the frequency of classical monocytes was significantly elevated in blood engrafted with CD34+ HSPCs from the WSD group (Figure 4G and Supplementary Figure 4I), while no changes were observed in T, B, or NK-cell populations (Supplementary Figures $\mathbf{4 G}$ and $\mathbf{4 H}$ ). In summary, this study demonstrates that WSD-induced alterations in phenotypic properties of FBM HSPCs persist even after transplanting into chow-fed recipient mice.

\section{Maternal WSD dampens fetal blood monocyte responses.}

We next asked if exposure to maternal WSD alters the frequencies of fetal blood immune cells (Figure 1A). Although we observed no changes in frequencies of blood monocytes and their major subsets (Figure 5A) or in lymphocyte subsets (Figure 5B-D), fewer monocytes within the peripheral blood mononuclear cell (PBMC) fraction responded to ex vivo LPS stimulation as measured by the frequency of IL-6/TNF $\alpha$ producing cells (Figure 5E) or the levels of IL-6/TNF $\alpha$ secreted following LPS stimulation (Figure 5F). Fetal PBMCs from the WSD group secreted significantly lower levels of chemokines (CCL5, CXCL10, CCL11, CXCL13) and factors associated with induction of adaptive immunity (IL-2, IFN $\gamma$, GZMB, IL-17) following LPS stimulation (Figure 5G). These studies demonstrate that maternal WSD alters the functional potential of fetal monocytes. 


\section{DISCUSSION}

Barker and colleagues, in their theory of the Developmental Origins of Health and Disease, proposed that both maternal overnutrition and undernutrition during pregnancy alter the offspring's subsequent cellular responses to the environment during childhood and adult life (Barker et al., 1989; Hales et al., 1991). Retrospective studies have demonstrated that pregravid obesity results in defects in cognitive development, cardiac abnormalities and metabolic syndrome in the offspring (Alfaradhi and Ozanne, 2011; Drake and Reynolds, 2010; Li et al., 2011; Oben et al., 2010). Several lines of evidence suggest that these developmental defects extend to the fetal immune system, manifesting as dampened anti-microbial defenses and aberrant autoimmune responses (Sureshchandra et al., 2019). For example, pups born to obese dams fed a HFD during gestation generated lower ovalbumin (OVA)-specific IgG, but higher OVAspecific IgE compared to pups born to dams fed a control diet (Odaka et al., 2010). Furthermore, pups from HFD-fed dams exhibited increased susceptibility to respiratory syncytial virus infection (Griffiths et al., 2016), greater mortality in an E.coli sepsis model, and higher bacterial burden after infection with methicillin-resistant Staphylococcus aureus (Myles et al., 2013). In contrast to the blunted antimicrobial responses, pups born to dams on HFD showed increased hyperreactivity to methacholine challenge (Griffiths et al., 2016) and were more likely to develop experimental autoimmune encephalitis following injection of myelin oligodendrocyte glycoprotein (Myles et al., 2013). Finally, ex vivo LPS stimulation of colonic lamina propria lymphocytes isolated from pups born to dams fed a HFD resulted in greater levels of IL-6, IL-1 3 , and IL-17, whereas LPS stimulation of splenocytes from the same animals generated decreased levels of TNF $\alpha$ and IL-6 compared to control groups, suggesting niche-dependent reprogramming of fetal myeloid cells (Myles et al., 2013).

We hypothesized that the immune cell defects in nonhuman primate fetal blood and tissues from offspring of moms consuming a WSD originate in precursor cell populations due to altered developmental programs. To test this, we leveraged the rhesus macaque model to study 
the effects of WSD-induced maternal obesity on FBM development and hematopoiesis. We report a positive correlation between maternal weight and fetal weight, in line with published human studies showing that excessive gestational weight gain in pregnant women is associated with increased fetal weight and increased neonatal adiposity (Barbour et al., 2018; Harmon et al., 2011; Rastogi et al., 2015; Shapiro et al., 2015; Suk et al., 2016). The present study also demonstrates the premature emergence of BM adipocytes and accelerated formation of the BM cavity during late gestation. The emergence of HSPCs in the FBM coincides with the development of the BM vascular system, bone ossification, and formation of the central marrow cavity in mice (Christensen et al., 2004; Coskun et al., 2014). The ability of HSPCs to home to FBM is regulated by emerging osteolineage cells (Christensen et al., 2004; Coskun et al., 2014). Maternal obesity, therefore, may exert distinct effects on fetal hematopoiesis via intrinsic (e.g., change in HSPC epigenome) and extrinsic (e.g., change in FBM microenvironment) mechanisms. We hypothesize that the premature maturation of the FBM niche results in an altered or suboptimal microenvironment for fetal HSPC differentiation, including a decrease in the number of supporting osteolineage cells and/or stromal mesenchymal stem cells (MSCs). Consistent with the latter idea, a reduction in the number of IL-7 producing MSCs has been shown to inhibit B-cell development and maturation in the murine BM (Zehentmeier and Pereira, 2019).

Focusing on the late stage of fetal development, we analyzed the FBM CD34+ HSPCs in GD130-135 fetuses of dams on a WSD or a chow diet (term is 165 days in rhesus macaques). scRNA-Seq revealed that maternal WSD increased the abundance of CLPs characterized by the altered B-cell transcriptional program, including a reduction in the expression of the B-cell development genes VPREB1 (Bauer et al., 1988), VPREB3 (Shirasawa et al., 1993), BST2 (Goto et al., 1994), and CD79a (Gold et al., 1991). Importantly, maternal WSD also reduced the expression of CLP genes encoding integrin ITGA6 (CD49f) (Krebsbach and Villa-Diaz, 2017; Notta et al., 2011; Qian et al., 2006) and sialomucin CD164 (Forde et al., 2007; Pellin et al., 2019; Zannettino et al., 1998), which are required for HSPC homing to the BM. Consistent with the idea 
of attenuated maturation of B-cell progenitors, our immunophenotypic analysis shows that maternal WSD reduces the frequencies of B-lymphocytes and NK-cells in the FBM. Interestingly, fetal blood B-cell frequencies remained unchanged with maternal WSD. This observation can be partially explained by enrichment of CLPs with B-cell potential in the FBM of the WSD group, suggesting a compensatory mechanism to maintain mature B-cell output.

We also observed an accelerated shift of myeloid progenitors towards more mature monocytes, with no apparent expansion of primitive HSCs. This observation is in line with previous studies in rodents, where maternal HFD promoted excess differentiation of myeloid and lymphoid lineages in early gestational fetal liver (Kamimae-Lanning et al., 2015). Interestingly, the observed accelerated differentiation trajectory did not alter the myeloid cell output either in the FBM or peripheral blood, in contrasts to previous rodent studies showing that HFD induces unbalanced hematopoiesis characterized by increased myeloid and reduced lymphoid output (Adler et al., 2014; Lee et al., 2018; Liu et al., 2018; Nagareddy et al., 2014). Furthermore, blood monocytes from fetuses from WSD group responded poorly to ex vivo LPS stimulation, confirming the previously reported dampened response by cord blood monocytes from babies born to obese mothers (Sureshchandra et al., 2017; Wilson et al., 2015) as well as splenic (Myles et al., 2013) and liver macrophages (Soderborg et al., 2018) in pups born to obese rodent dams. Interestingly, other studies have demonstrated hyper-inflammatory responses in BM-derived macrophages (Friedman et al., 2018) and microglia (Edlow et al., 2019) of offspring born to dams on HFD, suggesting a niche-specific impact on cellular rewiring. Indeed, our studies showed enhanced TLR2 cytokine responses by FBM stromal macrophages with WSD exposure (Supplementary Table S5). Heightened inflammatory responses by BM stromal macrophages could modulate the development of myeloid progenitors leading to "immune-tolerant phenotype" in mature monocytes. Recent studies from our and other laboratories have shown that BM adipocytes may potentiate myeloid-biased differentiation of HSPCs in adult rhesus macaques (Robino et al., 2020) 
and humans (Aguilar-Navarro et al., 2020). Future studies will identify adipocyte-derived molecules that control myelopoiesis.

To confirm if maternal WSD results in functional impairment of FBM HSPCs, we tested their regenerative and differentiation capacity in immunodeficient NSG mice. Our analysis demonstrated a significant reduction in HSPC homing to the BM and spleens of mice injected with fetal CD34+ cells from the WSD group. Additionally, frequencies of mature myeloid cells were diminished in the BM but enhanced in the blood and spleens, suggesting accelerated myeloid cell differentiation in extramedullary organs at the expense of BM hematopoiesis. Finally, we observed elevated rhesus macaque CD45+ cells in the blood of NSG mice injected with CD34+ cells from the WSD group, suggesting accelerated immune cell development and egress from the FBM. Taken as a whole, our study reports functional remodeling of FBM hematopoietic progenitors concomitant with the inadequate maturation state of the BM niche as a result of maternal obesity. This acceleration of FBM maturation may set a stage for altered or abnormal immune cell development, resulting in immunological abnormalities in the offspring.

While these findings are revealing in the context of developmental origins of immune dysfunction, our study was not able to capture multiple developmental stages/hematopoietic sites and the full breath of the effect of maternal WSD consumption on the cellular mechanisms that lead to altered HSPC programming. It is possible that the intersection of aberrant metabolic cues with dysregulated cytokine and inflammatory signaling pathways can affect epigenetic states of HSPCs, altering the timing/magnitude of gene expression programs and therefore lineage fate decisions in the FBM. Furthermore, repeated exposure to inflammatory signals in the FBM could serve as a 'first-stimulus' for progenitor cells, triggering pathways associated with innate immune tolerance/training. The extent of immune activation in the FBM, however, remains to be determined. Future studies will need to focus on the role of niche-specific signals in regulating both phenotype and functional responses in postnatal tissue resident cells and if/how dietary 
bioRxiv preprint doi: https://doi.org/10.1101/2021.04.26.441556; this version posted April 27, 2021. The copyright holder for this preprint (which was not certified by peer review) is the author/funder. All rights reserved. No reuse allowed without permission.

intervention before/during gestation ablates functional defects observed in fetal hematopoietic niche. 


\section{MATERIALS AND METHODS}

\section{Animal procedures}

All animal procedures were approved by the Oregon National Primate Research Center (ONPRC) Institutional Animal Care and Use Committee and comply with the Animal Welfare Act and the APA Guidelines for Ethical Conduct in the Care and Use of Nonhuman Animals in Research. Dietary interventions, animal care, and fertility trial procedures have been previously reported (Bishop et al., 2018; Bishop et al., 2021; True et al., 2017; Varlamov et al., 2017)

C-section. Food was withheld for approximately 12 hours prior to the procedure. Water was not withheld. Animals were sedated with $8-20 \mathrm{mg} / \mathrm{kg}$ ketamine. Following sedation, a local block consisting of $0.8 \mathrm{ml}$ bupivicaine $(0.5 \%)$ combined with $0.2 \mathrm{ml}$ lidocaine $(1 \%)$ with epinephrine was placed intradermally at the incision site. Once the intravenous catheter was placed, the animal received $0.025-0.2 \mathrm{mg} / \mathrm{kg}$ hydromorphone intravenously. Animals were endotracheally intubated with an appropriate sized endotracheal tubes and general anesthesia was induced with 3\% Isoflurane for 2-3 minutes or until the animal began to lose reflexes and heart rate slowed. Inhalant anesthesia was maintained at $1-2 \%$ isoflurane which was adjusted based on the physiologic parameters of the animal. Inhalant anesthetics were combined with $100 \%$ oxygen administered at a rate of $1-1.5 \mathrm{~L} / \mathrm{min}$. All surgical procedures were conducted by trained ONPRC Surgical Services Unit personnel under the supervision of surgical veterinarians in dedicated surgical facilities using aseptic techniques and comprehensive physiological monitoring. All animals had 22-gauge cephalic catheters placed and were intubated in dorsal recumbency with an appropriate size endotracheal tubes. Animals received intravenous fluids (generally Lactated Ringers Solution) at $10 \mathrm{ml} / \mathrm{kg} / \mathrm{hour}$; fluids were increased based on hydration status of animal or due to blood loss during surgery. The animal was positioned in dorsal recumbency, followed by sterile prep and draping. The abdomen was entered via 10-cm linear ventral midline laparotomy, followed by delivery and draping of the gravid uterus with moistened laparotomy sponges. The fetus was balloted to the fundic region, and a transverse hysterotomy 
was made and delivery of the fetus was completed. Fetuses were sedated with $10-20 \mathrm{mg} / \mathrm{kg}$ of ketamine and transported to the ONPRC Pathology Services Unit. Fetuses were anesthetized with sodium pentobarbital administered via the umbilical vessels at $25 \mathrm{mg} / \mathrm{kg}$ and euthanized by exsanguination. Fetal bone samples were collected and processed within 15 minutes of exsanguination.

\section{Cell isolation}

For FBM cell isolation, both fetal rhesus macaque tibias were crushed manually with a ceramic mortar and pestle and the cell suspension was filtered through a $30-\mu$ m nylon cell strainer (Thermo Fisher Scientific, Waltham, MA, USA) in $20 \mathrm{ml}$ ice-cold X-Vivo media (Lonza, Basel, Switzerland). FBM mononuclear cells were isolated by centrifugation for 30 minutes at room temperature on a Ficoll gradient (StemCell Technologies, Vancouver, BC, Canada) and red blood cells were removed using the RBC lysis buffer (StemCell Technologies). Mononuclear cells were washed three times with PBS and CD34+ cells were isolated as described (Robino et al., 2020) using rhesus macaque-specific antibodies to CD34 (clone 563; BD Pharmingen, San Jose, CA, USA) and the anti-PE MACS magnetic bead system (Miltenyi Biotec, Bergisch Gladbach, Germany) with the supplied reagents. CD34+ HSPC and CD34- flow-through cell fractions were cryopreserved in several aliquots using CryoStor CS10 (Stem Cells Technologies).

\section{Engraftment of HSPCs in NSG mice}

The procedures were performed on adult 4-week old female NSG mice (Jackson Laboratory, Bar Harbor, ME, USA; Strain: 005557 NOD.Cg-Prkdc II2rg/SzJ) of at least 15 grams body weight. The NSG mice were maintained on regular rodent chow. Neither food nor water were withheld. Drinking water was supplemented with prophylactic antibiotics (1.2 mM Neomycin trisulfate and $0.13 \mathrm{mM}$ Polymyxin B sulfate). To minimize non-hematopoietic toxicity, a non-lethal dose of 200 cGy was administered to NSG mice. BM cells were replenished with 
donor cells injected within 4 hours after irradiation. For retroorbital injection of HSPCs, the mouse was anesthetized using inhaled isoflurane at $4 \%$ for induction and $1.5-2 \%$ during the procedure, delivered in $100 \%$ oxygen at a rate of $1.5-2 \mathrm{~L} /$ minute. The mouse was kept warm on a warming pad. A sterile 27 -gauge needle attached to a sterile $1.0 \mathrm{ml}$ syringe was used for the injection of CD34+ cells. After a surgical plane of anesthesia has been achieved, the needle was inserted between the medial canthus and the globe. The tip of the needle was directed at the rostrodorsal aspect of the orbit and gently advanced toward the ophthalmic plexus. A volume of up to $200 \mu \mathrm{l}$ was injected. When the injection was completed, the needle was removed and the mouse was observed for bleeding at the injection site. For blood collection, the mouse was manually restrained in the operator's non-dominant hand by securing the loose skin on the dorsal head, neck, and thorax with the thumb and index finger. The puncture site was identified $2 \mathrm{~mm}$ from the "dimple" near the oral commissure, along an imaginary line from the dimple to the pinna. A lancet of appropriate size is used to puncture the skin and vein. Blood was allowed to drip into a collection vial filled with $5 \mathrm{ml}$ EDTA-PBS solution. When bleeding has stopped, manual restraint was released, providing hemostasis. The mouse was placed back in the cage and observed for additional bleeding as well as for signs of respiratory distress. Blood collection was performed once a month.

\section{Bone histology}

Histological analyses were performed on paraffin-embedded sections. Specifically, fetal femurs were fixed in $4 \%$ PFA for 24 hours at $4^{\circ} \mathrm{C}$, decalcified in $0.5 \mathrm{M}$ EDTA for 4-7 days, and embedded in paraffin. Adjacent 5- $\mu \mathrm{m}$ paraffin sections were stained with hematoxylin and eosin to evaluate bone morphology and adipogenesis.

\section{Flow cytometry}


RBC-free cell pellets were processed and cryopreserved in 4-5 aliquots as described (Robino et al., 2020; Varlamov et al., 2020). Cryopreserved cells, including tibial FBM and PBMCs, were analyzed by flow cytometry using monoclonal antibodies validated for binding to rhesus macaque proteins (Burwitz et al., 2014) and lymphoid-specific and myeloid-specific gating strategies. For FBM phenotyping, $5 \times 10^{5}$ cells were stained with antibodies to CD45 (D058-1283, Biolegend), CD3 (clone SP34, BD Pharmingen), CD20 (clone 2H7, Biolegend), CD8 (clone SK1, Biolegend), CD14 (clone M5E2, Biolegend), CD16 (clone 3G8, Biolegend), HLA-DR (clone L243, Biolegend), CD11b (clone ICRF44, Biolegend), CD163 (clone GHI/61, Biolegend), Mac387 (AB7429, Abcam). For blood phenotyping, 5x105 freshly thawed PBMCs were stained with CD3 (clone SP34, BD Pharmingen), CD8b (clone 2ST8.5H7, Beckman Coulter), CD20 (clone 2H7, Biolegend), CD14 (clone M5E2, Biolegend), HLA-DR (clone L243, Biolegend), CD11c (clone 3.9, Biolegend), and CD123 (clone 6H6, Biolegend), and CD16 (clone 3G8, Biolegend). Cells were incubated at 4C for 30 minutes, washed twice in FACS buffer (1X PBS with $2 \%$ FBS and $1 \mathrm{mM}$ EDTA) and acquired on Attune NxT (ThermoFisher).

\section{$\underline{\text { TLR stimulation assays }}$}

FBM cells $\left(5 \times 10^{5}\right)$ were stimulated with LPS (TLR2/4 agonist $\left.1 \mu \mathrm{g} / \mathrm{ml}\right)$, Pam3CSK4 (TLR1/2 agonist, $1 \mu \mathrm{g} / \mathrm{ml}$ ) or HKLM (TLR2 agonist, $10^{8}$ particles $/ \mathrm{ml}$ ) for 8 hours in the presence of $5 \mu \mathrm{g} / \mathrm{ml}$ brefeldin A. Cells were stained with antibodies to CD11b, HLA-DR and CD163 to delineate monocytes/macrophages. Following surface staining, cells were permeabilized and stained with monoclonal antibodies to TNFa. Samples were washed in FACS buffer and analyzed on an LSRII flow cytometer (BD Biosciences, San Jose, CA). Data were acquired using BD FACS Diva (BD Biosciences) and post-analysis performed using FlowJo v10 (Tree Star, Ashland, OR) and Prism v6 (GraphPad Software, San Diego, CA). 5×105 fetal PBMC were stimulated with 1 $\mu \mathrm{g} / \mathrm{mL}$ LPS for 16 hours in the presence/absence of Brefeldin A. For samples with Golgi blocking, 
cells were surface-stained with CD14 (clone M5E2, Biolegend), CD16 (clone 3G8, Biolegend), and HLA-DR (clone L243, Biolegend), then permeabilized and stained intracellularly with TNF $\alpha$ (clone MAb11, Invitrogen) and IL6 (MQ2-6A3, BD Biosciences). Samples were washed in FACS buffer and analyzed on the Attune NxT flow cytometry (ThermoFisher). Supernatants were collected from samples with no BFA and stored at $-80^{\circ} \mathrm{C}$ until further analysis.

\section{Luminex cytokine assays}

Cytokines, chemokines, and growth factors in undiluted cell culture supernatants were measured using a 37-plex NHP XL Cytokine Luminex Performance kit (R \& D Systems, Minneapolis MN). Samples were run in duplicates on a Magpix instrument (Luminex, Austin TX). Data were fit using a 5P-logistic regression on the XPONENT software. Values below the limit of detection were designated as half of the lowest limit.

\section{FACS sorting and scRNA-Sequencing}

Freshly thawed CD34+ HSPCs were enriched for live cells by FACS using SYTOX Blue dead cell stain (ThermoFisher) on a BD FACS Aria Fusion cell sorter. Cells were sorted into RPMI containing $30 \%$ FBS, $5 \%$ glutamine, and antibiotics. Cells were then counted in triplicates on a TC20 Automated Cell Counter (Bio-Rad), washed and resuspended in 1X PBS with 0.04\% BSA to a final concentration of 1200 cells/ $\mu \mathrm{L}$. Single cell suspensions were then immediately loaded on a 10X Genomics Chromium Controller with a loading target of 17,600 cells. Libraries were generated using the 3' V3 chemistry per the manufacturer's instructions (10X Genomics, Pleasanton, CA) and sequenced on an Illumina NovaSeq with a sequencing target of 30,000 reads per cell.

\section{Single cell RNA-Seq data analysis}


Raw reads were aligned and quantified using the Cell Ranger's count function (version 4.0, 10X Genomics) against the Macaca mulatta reference genome (MMul10) using the STAR aligner. Downstream processing of aligned reads was performed using Seurat (version 3.2.2). Droplets with ambient RNA (cells with fewer than 400 detected genes), potential doublets (cells with more than 4000 detected genes), and dying cells (cells with more than $20 \%$ total mitochondrial gene expression) were excluded during the initial quality control. To rule out biases in different stages of cell cycle, cells were then scored for S or G2/M phase using a catalog of canonical markers (Kowalczyk et al., 2015). Cells with high ribosomal gene expression ( $\geq 30$ pc of total gene expression) were further excluded before integration. Data objects from the two groups were integrated using Seurat's IntegrateData function (Stuart et al., 2019). Data normalization and variance stabilization were performed using SCTransform function using a regularized negative binomial regression to correct for differential effects of mitochondrial and ribosomal gene expression profiles. Dimension reduction was performed using the RunPCA function to obtain the first 30 principal components followed by clustering using the FindClusters function in Seurat. Clusters were visualized using UMAP algorithm as implemented by Seurat's runUMAP function. Cell types were assigned to individual clusters using FindMarkers function with a fold-change cutoff of at least 0.4 and using a known catalog of well-characterized scRNA markers for HSCs (Supplementary Table S5) (Pellin et al., 2019).

\section{Pseudo-temporal and differential expression analyses}

Hematopoietic lineages and developmental pseudo time of myeloid cells was inferred by Slingshot. The annotated clusters inferred by KNN graph were used as input for Slingshot. Differential expression analysis of the chow and WSD groups within each cell type was performed using the non-parametric Wilcoxon rank sum test in Seurat. Genes with at least a 0.4-fold change $\left(\log _{2}\right.$ scale) and with an adjusted $p$-value $\leq 0.05$ were considered significant. Pathway analysis of differential signatures was performed using Enrichr. For module scoring, we compared gene 
signatures and pathways from KEGG (https://www.genome.jp/kegg/pathway.html) in subpopulations using Seurat's AddModuleScore function. Group differences were compared using unpaired t-test followed by Welch's correction.

\section{Statistical analyses}

All statistical analyses were conducted in Prism 8 (GraphPad). All definitive outliers in twoway and four-way comparisons were identified using ROUT analysis $(Q=0.1 \%)$. Data was then tested for normality using the Shapiro-Wilk test (alpha=0.05). If data were normally distributed across all groups, differences with were tested using ordinary one-way ANOVA with unmatched samples. Multiple comparisons were corrected using the Holm-Sidak test adjusting the familywise significance and confidence level at 0.05 . If Gaussian assumption was not satisfied, differences were tested using a Kruskal-Wallis test (alpha=0.05) followed by Dunn's multiple hypothesis correction test. Differences in normally distributed two groups were tested using an unpaired t-test followed by Welch's correction (assuming different standard deviations). Two group comparisons that failed normality tests were tested for differences using a Mann-Whitney test. For subsets of cells within a sample, differences were tested using paired t-tests.

We conducted a correlation analysis between maternal and petal parameters by calculating the Pearson correlation coefficients and their associated p-values. The p-values were then adjusted using Benjamini-Hochberg procedure to control the false discovery rate (FDR) below 0.05 for selected maternal fetal parameters. This method was implemented in $\mathrm{R}$ using the "p.adjust" function.

Luminex data were tested for normality using the Shapiro-Wilk test. Differences in protein in supernatants were tested using ordinary one-way ANOVA followed by a Holm-Sidak multiple comparison test. 


\section{SUPPLEMENTARY TABLES}

Supplementary Table S1. Maternal parameters prior to and during pregnancy.

Supplementary Table S2. Fetal and maternal body composition parameters and glucose levels at C-section.

Supplementary Table S3. Correlations between maternal and fetal parameters.

Supplementary Table S4. Single cell markers used for progenitor cluster identification.

Supplementary Table S5. Differentially expressed genes with maternal WSD identified within major CD34+ cell clusters. 


\section{ACKNOWLEDGEMENTS}

We thank the ONPRC Integrated Pathology Core for help with microscopy, the ONPRC Division of Comparative Medicine for help with bone sectioning, and the ONPRC Flow Cytometry Core for help with flow-cytometry analyses. We thank Dr. Lauren Drew Martin for help with blood collections. We thank the head of the Small Lab Animal Unit Kati Marshall for help in animal handling and transplantation. We thank Dr. Jennifer Atwood (UCI Flow Cytometry Core) for assistance with cell sorting and Dr. Melanie Oakes (UCI GHTF) for assistance with library preparation and sequencing of $10 \mathrm{X}$ samples. This study was supported by NIH grants P50 HD071836 to CTR and JDH, P51 OD01192 for operation of the Oregon National Primate Research Center, and NIH grants 1R01AI142841 and 1R01AI145910 to IM.

\section{AUTHOR CONTRIBUTIONS}

scRNA-Seq assay and bioinformatic analysis were generated by SS. HSPC transplantation and cell isolation in NSG mice were performed by OV, JJR and DG. Immunophenotyping experiments were performed by BJB, SS, OV, and JJR. Flow cytometry data were analyzed by IM, OV, SS, DG and BJB. Fetal collection was performed by DT, JJR and OV. Statistical analysis was performed by OV, SS, BJB, and WS. The paper was written by OV, SS, and IM. OV, JDH, and CTR supervised the study and edited the manuscript.

\section{DECLARATION OF INTERESTS}

Authors have nothing to declare

\section{DATA AVAILABILITY}

Gene expression data are available at the NCBI SRA data repository under Accession Number PRJNA723061. 


\section{FIGURE LEGENDS}

Figure 1: Animal model and experimental design. (A) Experimental Design - Tibias from fetuses (gestational day 130-135) were isolated from dams on chow $(n=9)$ and on WSD $(n=4)$. CD34+ cells were isolated from bone marrow using magnetic bead enrichment and profiled using scRNA-Seq and assessed for engraftment and regenerative capacity. FBM mononuclear cells from the CD34-negative fraction and PBMCs were phenotyped using flow cytometry and functional responses to TLR stimulation were tested. (B) Maternal weight, (C) body fat, (D) fetal weight, and $(E)$ crown-rump length were determined at C-section. (F) Pearson correlations between maternal and fetal weights; $r$-value and p-value are indicated. (G) Representative H\&E staining of proximal fetal femurs from the control (top) and WSD groups (bottom); the outlined areas are enlarged (right); b, bone; a, adipocytes; s, central sinus; scale bars=1 mm; control femur does not contain the BM cavity, but forms ossified trabeculae (pink, arrow); WSD femur forms the central cavity filled with red marrow (violet, arrow) and adipocytes. $(H)$ FBM adipocyte content in fetal femur. (I) Comparison of CD34+ cell frequencies within FBM. Bars are means \pm $S D ;{ }^{*} p<0.05 ;{ }^{* *} p<0.01$.

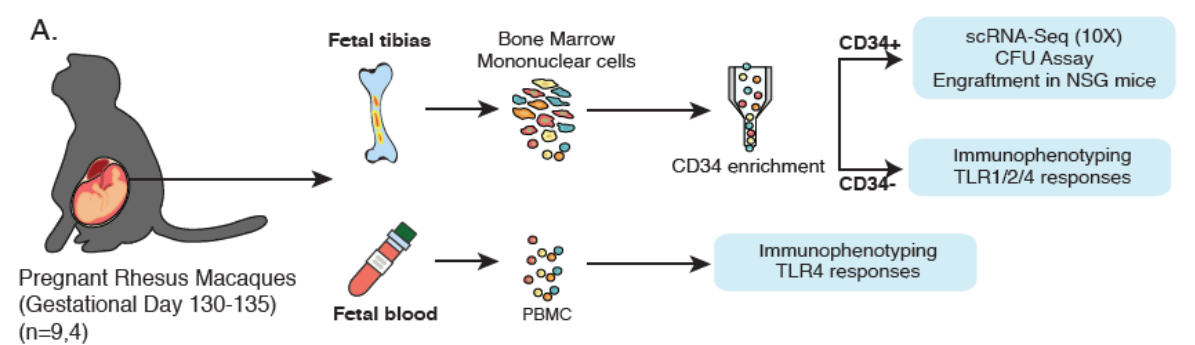

B.

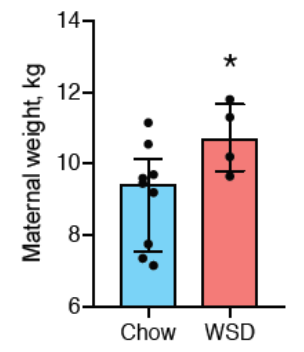

C.

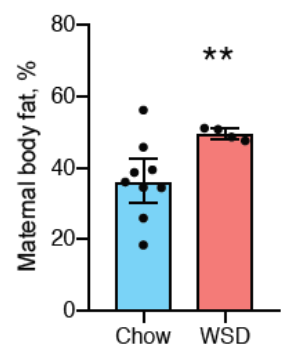

D.

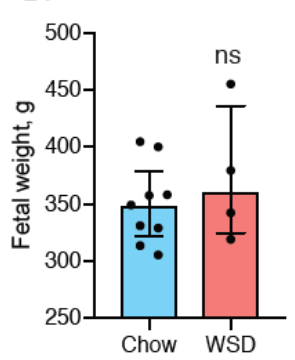

E.

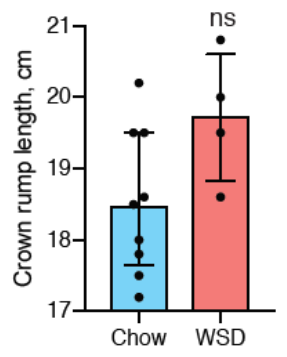

F

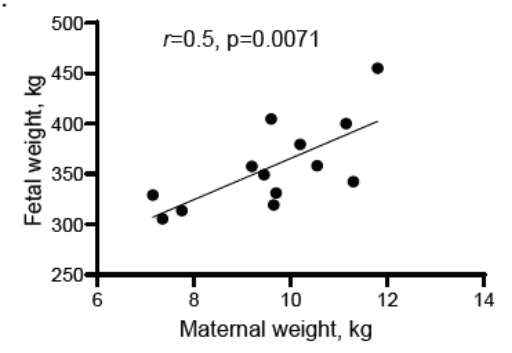

G.

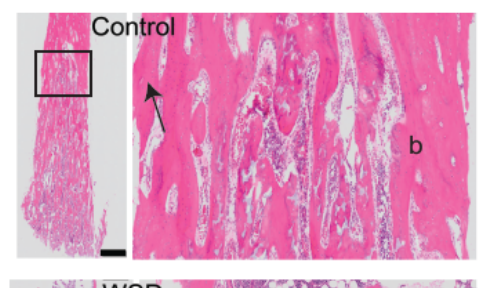

$\mathrm{H}$.

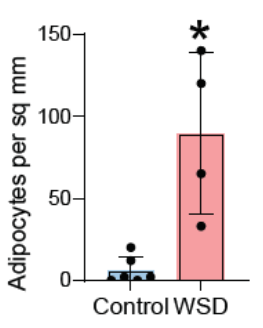

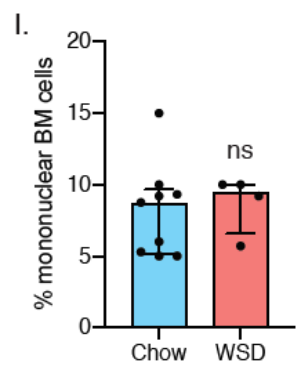

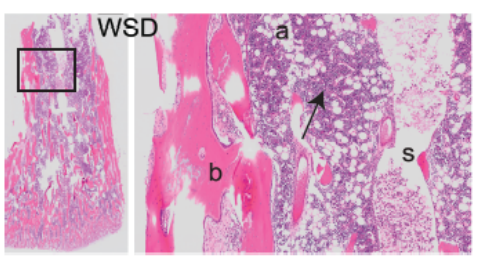


Figure 2: Maternal exposure to WSD induces phenotypic changes in FBM hematopoietic progenitors and lowers the frequencies of BM-derived lymphoid cells. (A) Uniform Manifold Approximation and Projection (UMAP) was used to group single-cell clusters after dimension reduction of sorted CD34+ cells ( $n=3 /$ group, pooled). (B) Heatmap representation of the top 20 markers that were used for identification of individual UMAP clusters. Only a handful of markers are annotated for visualization of the data. (C) Stacked bar graphs compare relative proportions of individual clusters in control and WSD groups. (D) Stacked bar graphs compare individual CLP clusters as a fraction of CD34+ cells. (E) Pathway analysis shows up-regulated (bars in red) and down-regulated (bars in blue) genes in CLPs from WSD group identified using Enrichr. (F) Violin plot compares module scores for B-cell receptor (BCR) signaling within the CLP clusters. (G) Bcell frequencies in the CD34-CD45+ fraction of the FBM. $(\mathrm{H})$ Violin plot compares module scores for T-cell receptor (TCR) signaling within the CLP clusters. (I) T-cell and (J) NK-cell frequencies in the CD34-CD45+ fraction of the FBM. Each data point represents one fetus. Bars are means \pm SD. All two-group comparisons were tested for statistical differences using unpaired t-tests. $;^{*} p<0.05 ;{ }^{* *} p<0.01 ;{ }^{* * *} p<0.001 ;{ }^{* * * *} p<0.0001$.

A.

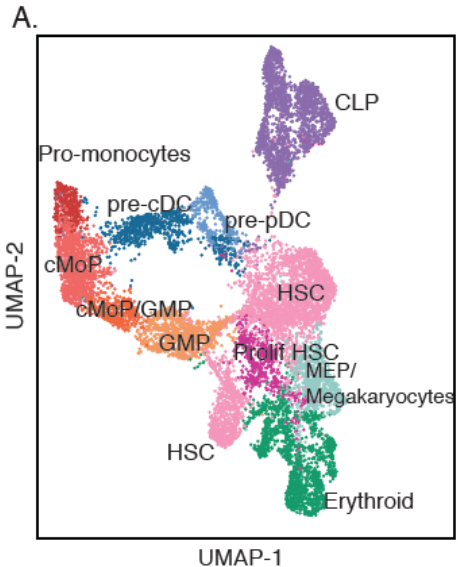

E.

Differential analysis of CLP clusters


B.

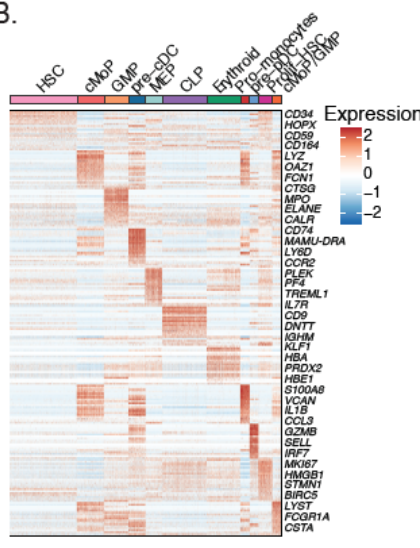

C.

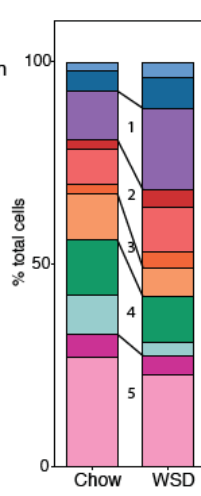

D.

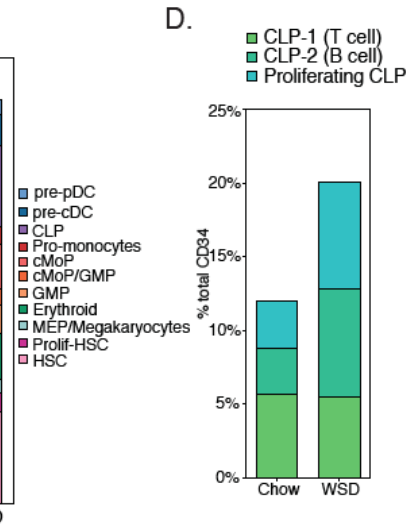

F.

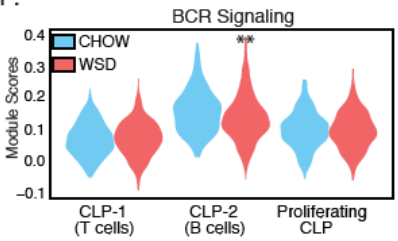

G

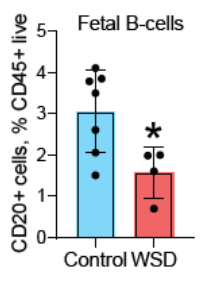

$\mathrm{H}$.

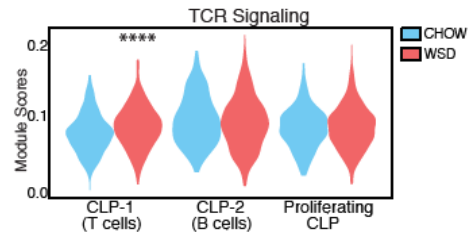

I.

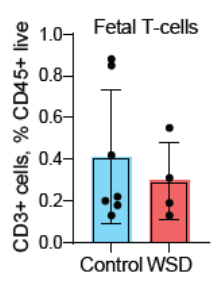

$\mathrm{J}$.

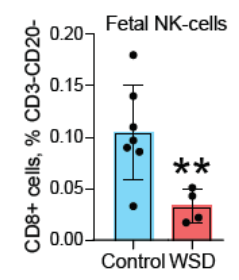


Figure 3: Maternal exposure to WSD accelerates myeloid precursor development and heightens pro-inflammatory responses in stromal macrophages in FBM. (A) Unsupervised pseudo-temporal analysis of myeloid clusters using Slingshot. Arrow suggests the most probable direction of developmental trajectory. (B) Waffle charts representing percentages of GMP, GMP/cMoP, cMoP, and pro-monocytes within FBM myeloid cell precursors in control and WSD groups. (C) Box plots comparing cellular states of HSCs and myeloid precursors in relation to their position on developmental pseudotime. (D) Pathway analysis of upregulated (bars in green) and down-regulated (bars in yellow) genes by maternal WSD in HSC clusters identified using Enrichr. (E) Violin plots comparing module scores for cytokine receptor signaling and chemokine signaling within myeloid cell precursors. (F) Bar graphs comparing median fluorescence intensities of TLR4, CD86, and HLA-DR on gated monocytes (bottom panel) and macrophages (top panel) in the FBM. (G) Dot plots comparing TNF $\alpha$ responses following TLR ligand-stimulated FBM macrophages from 4 control and 4 WSD fetuses. Each data point shows the $\%$ of TNF $\alpha^{+}$ cells within the CD11b+HLA-DRlo/-CD163+ gate; horizontal bars are means. $(H)$ Correlation between maternal adiposity (measured before pregnancy) and frequencies of TNF $\alpha+F B M$ macrophages/monocytes. Pearson correlation coefficient $(r)$ and $p$-value are indicated. All twogroup comparisons were tested for statistical differences using unpaired t-tests; ${ }^{*} p<0.05$; ${ }^{* *} p<0.01 ;{ }^{* * *} p<0.001 ;{ }^{* * *} p<0.0001$.



E.
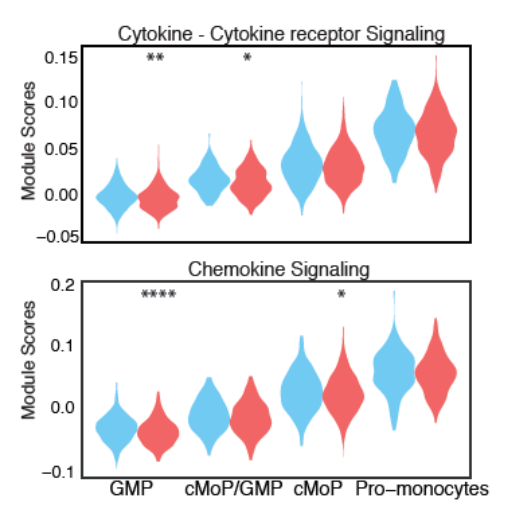

.
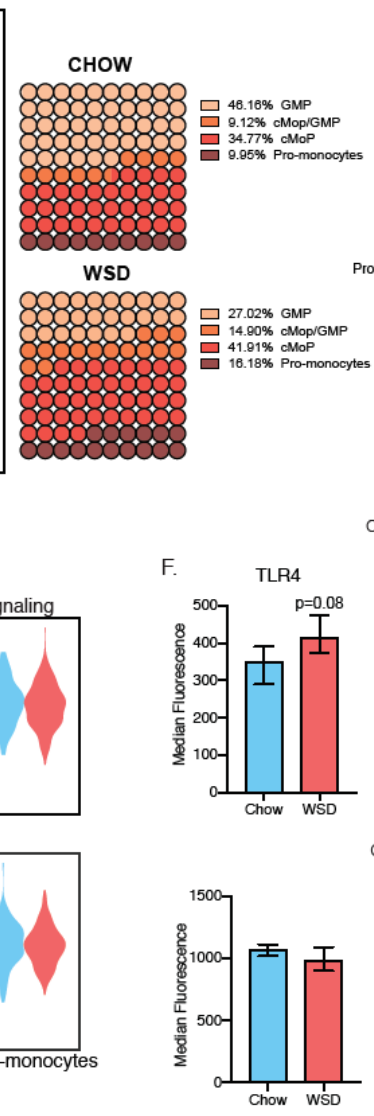

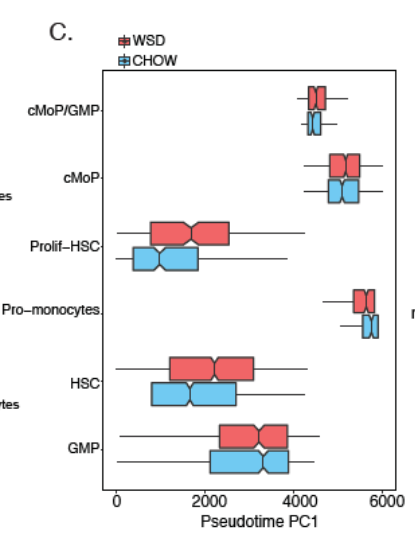

CD14+ HLA-DRlow

(Macrophages)
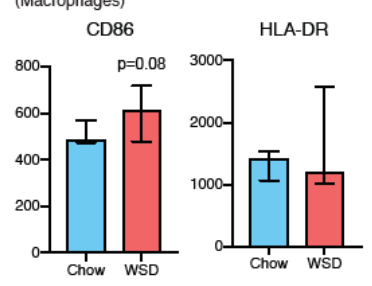

CD14+ HLA-DRhigh
(Monocytes)


D.

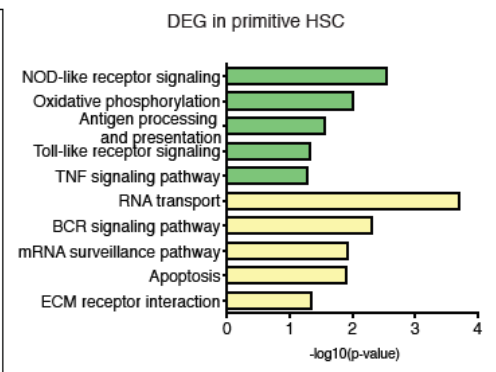

G.

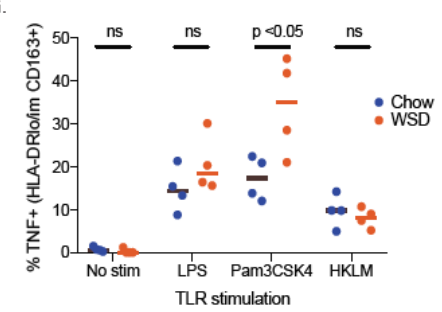

$\mathrm{H}$

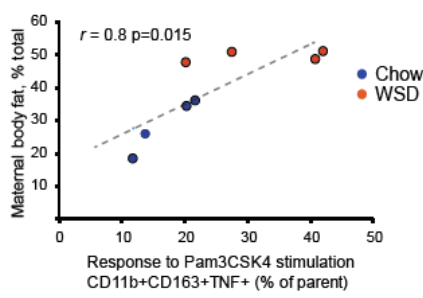


Figure 4: Maternal WSD alters the regenerative ability of HSPCs and reduces myeloid cell reconstitution in immunodeficient mice. (A) Outline of engraftment experiments. (B) Engraftment efficiencies of isolated rhesus FBM CD34+ cells in NSG mice. (C) Relative frequencies of rhesus CD34+ cells in the bone marrow and spleen of NSG mice. (D) Frequencies of rhesus myeloid cells $(C D 11 b+C D 14+)$ and recently infiltrating monocytes/macrophages $(C D 11 b+$ Mac387+) in the NSG mouse BM. (E-F) Bar graphs comparing frequencies of $(E)$ mature lymphoid cells and (F) infiltrating macrophages in spleens isolated from NSG mice. (G) Relative frequencies of monocyte subsets in the blood from NSG mice. Each data point represents one NSG mouse; bars are means \pm SD, t-test, ${ }^{*} p<0.05,{ }^{* *} p<0.01,{ }^{* * *} p<0.001,{ }^{* * *} p<0.0001$, NS, not significant.

A.

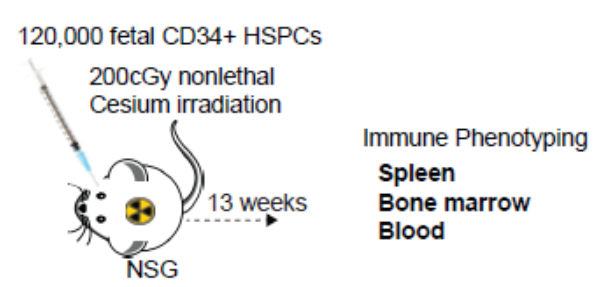

Bone marrow

D.
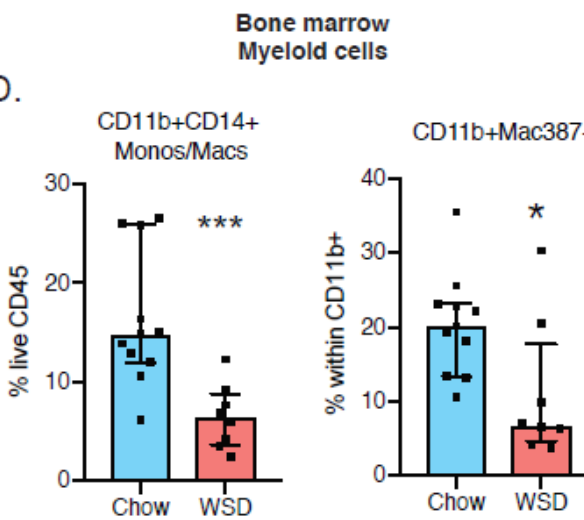

F. $\quad \begin{gathered}\text { Splenlc } \\ \text { Macrophages }\end{gathered}$

$\mathrm{CD} 11 \mathrm{~b}+\mathrm{Mac} 387+$

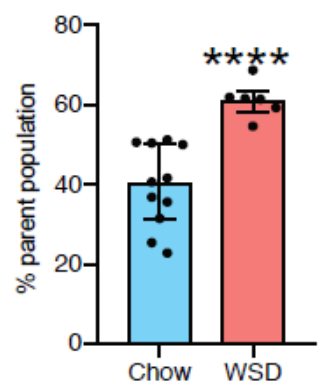

CD11b+Mac387+

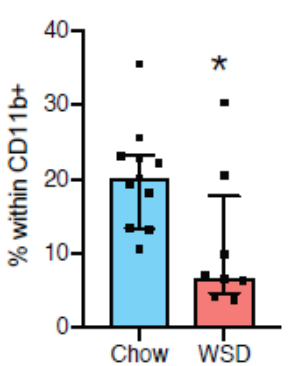

G.
B.

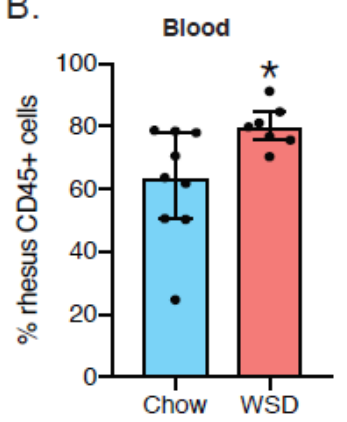

E.
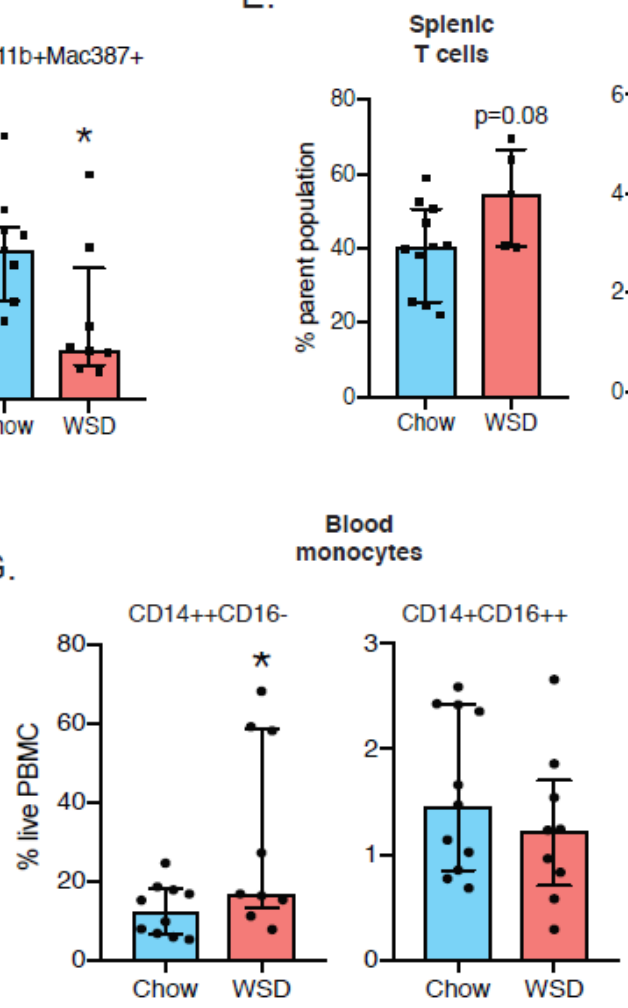

C.
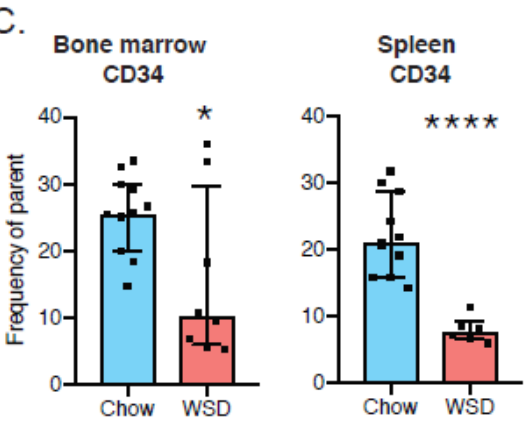




Figure 5: Maternal WSD attenuates fetal blood monocyte activation in response to bacterial lipopolysaccharide.

(A-D) Relative frequencies of $(A)$ monocytes and its subsets, $(B)$ T-cell subsets, $(C)$ B-cells, and (D) NK-cells in fetal peripheral blood. (E) Dot plot comparing TNF $\alpha$ and IL6-producing monocytes in response to a 16-hour-long LPS stimulation. (F) Principal Component Analysis (PCA) of 37 immune mediators released by fetal PBMCs in the presence/absence of LPS as measured by Luminex. (G) Heatmap of median cytokine and chemokine levels $(\mathrm{pg} / \mathrm{mL})$ secreted by fetal PBMC in the presence/absence of LPS.

A.

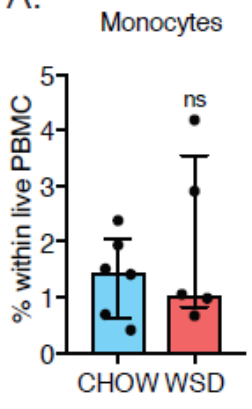

Classical Monos

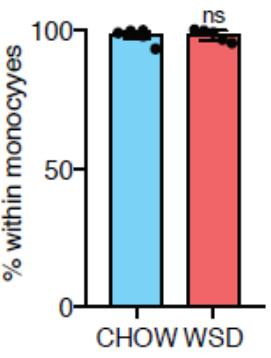

Non-classical

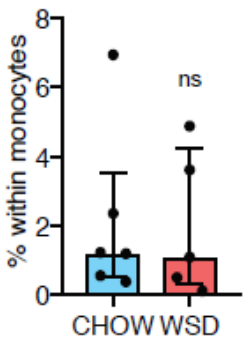

B



CD8 T cells

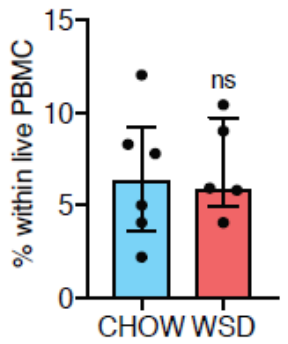

C.

B cells

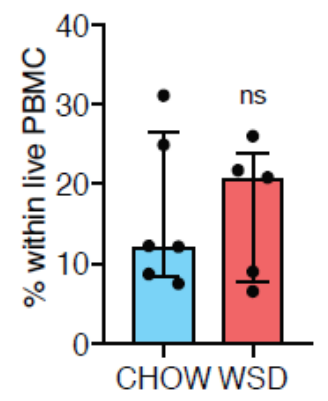

D.

NK cells

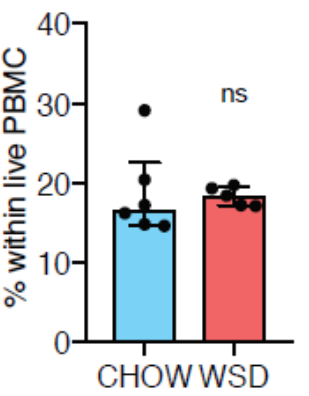

E. Chow

- WSD

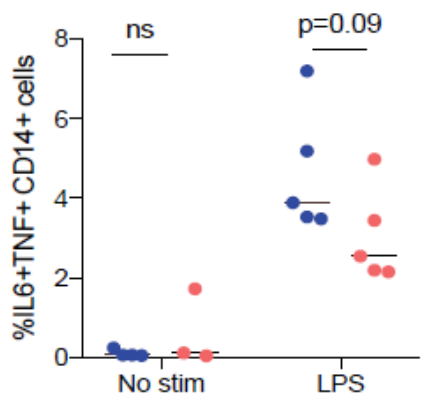

F.

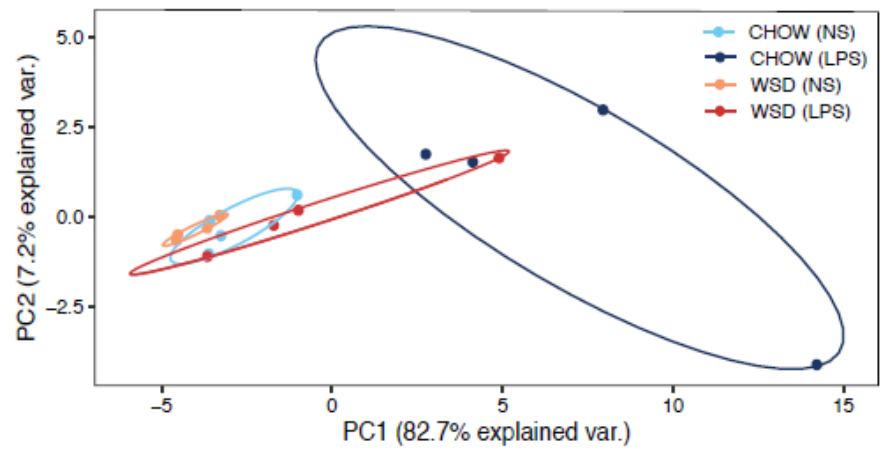

G. -1

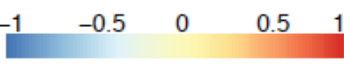




\section{REFERENCES}

Adler, B.J., Green, D.E., Pagnotti, G.M., Chan, M.E., and Rubin, C.T. (2014). High fat diet rapidly suppresses $B$ lymphopoiesis by disrupting the supportive capacity of the bone marrow niche. PLoS One 9, e90639.

Aguilar-Navarro, A.G., Meza-Leon, B., Gratzinger, D., Juarez-Aguilar, F.G., Chang, Q., Ornatsky, O., Tsui, H., Esquivel-Gomez, R., Hernandez-Ramirez, A., Xie, S.Z., et al. (2020). Human Aging Alters the Spatial Organization between CD34+ Hematopoietic Cells and Adipocytes in Bone Marrow. Stem Cell Reports 15, 317-325.

Alfaradhi, M.Z., and Ozanne, S.E. (2011). Developmental programming in response to maternal overnutrition. Front Genet 2, 27.

Barbour, L.A., Farabi, S.S., Friedman, J.E., Hirsch, N.M., Reece, M.S., Van Pelt, R.E., and Hernandez, T.L. (2018). Postprandial Triglycerides Predict Newborn Fat More Strongly than Glucose in Women with Obesity in Early Pregnancy. Obesity (Silver Spring) 26, 1347-1356. Barker, D.J., Osmond, C., Golding, J., Kuh, D., and Wadsworth, M.E. (1989). Growth in utero, blood pressure in childhood and adult life, and mortality from cardiovascular disease. BMJ 298, 564-567.

Bauer, S.R., Kudo, A., and Melchers, F. (1988). Structure and pre-B lymphocyte restricted expression of the VpreB in humans and conservation of its structure in other mammalian species. EMBO J 7, 111-116.

Bishop, C.V., Mishler, E.C., Takahashi, D.L., Reiter, T.E., Bond, K.R., True, C.A., Slayden, O.D., and Stouffer, R.L. (2018). Chronic hyperandrogenemia in the presence and absence of a western-style diet impairs ovarian and uterine structure/function in young adult rhesus monkeys. Hum Reprod 33, 128-139.

Bishop, C.V., Takahashi, D., Mishler, E., Slayden, O.D., Roberts, C.T., Hennebold, J., and True, C. (2021). Individual and combined effects of 5-year exposure to hyperandrogenemia and 
Western-style diet on metabolism and reproduction in female rhesus macaques. Hum Reprod $36,444-454$.

Bowie, M.B., Kent, D.G., Dykstra, B., McKnight, K.D., McCaffrey, L., Hoodless, P.A., and Eaves, C.J. (2007). Identification of a new intrinsically timed developmental checkpoint that reprograms key hematopoietic stem cell properties. Proc Natl Acad Sci U S A 104, 5878-5882.

Burwitz, B.J., Reed, J.S., Hammond, K.B., Ohme, M.A., Planer, S.L., Legasse, A.W., Ericsen, A.J., Richter, Y., Golomb, G., and Sacha, J.B. (2014). Technical advance: liposomal alendronate depletes monocytes and macrophages in the nonhuman primate model of human disease. J Leukoc Biol 96, 491-501.

Carbone, L., Davis, B.A., Fei, S.S., White, A., Nevonen, K.A., Takahashi, D., Vinson, A., True, C., Roberts, C.T., Jr., and Varlamov, O. (2019). Synergistic Effects of Hyperandrogenemia and Obesogenic Western-style Diet on Transcription and DNA Methylation in Visceral Adipose Tissue of Nonhuman Primates. Sci Rep 9, 19232.

Cedar, H., and Bergman, Y. (2011). Epigenetics of haematopoietic cell development. Nat Rev Immunol 11, 478-488.

Christensen, J.L., Wright, D.E., Wagers, A.J., and Weissman, I.L. (2004). Circulation and chemotaxis of fetal hematopoietic stem cells. PLoS Biol 2, E75.

Coskun, S., Chao, H., Vasavada, H., Heydari, K., Gonzales, N., Zhou, X., de Crombrugghe, B., and Hirschi, K.K. (2014). Development of the fetal bone marrow niche and regulation of HSC quiescence and homing ability by emerging osteolineage cells. Cell Rep 9, 581-590.

Drake, A.J., and Reynolds, R.M. (2010). Impact of maternal obesity on offspring obesity and cardiometabolic disease risk. Reproduction 140, 387-398.

Edlow, A.G., Glass, R.M., Smith, C.J., Tran, P.K., James, K., and Bilbo, S. (2019). Placental Macrophages: A Window Into Fetal Microglial Function in Maternal Obesity. Int J Dev Neurosci 77, 60-68. 
Forde, S., Tye, B.J., Newey, S.E., Roubelakis, M., Smythe, J., McGuckin, C.P., Pettengell, R., and Watt, S.M. (2007). Endolyn (CD164) modulates the CXCL12-mediated migration of umbilical cord blood CD133+ cells. Blood 109, 1825-1833.

Friedman, J.E. (2018). Developmental Programming of Obesity and Diabetes in Mouse, Monkey, and Man in 2018: Where Are We Headed? Diabetes 67, 2137-2151.

Friedman, J.E., Dobrinskikh, E., Alfonso-Garcia, A., Fast, A., Janssen, R.C., Soderborg, T.K., Anderson, A.L., Reisz, J.A., D'Alessandro, A., Frank, D.N., et al. (2018). Pyrroloquinoline quinone prevents developmental programming of microbial dysbiosis and macrophage polarization to attenuate liver fibrosis in offspring of obese mice. Hepatol Commun 2, 313-328. Gao, X., Xu, C., Asada, N., and Frenette, P.S. (2018). The hematopoietic stem cell niche: from embryo to adult. Development 145.

Gold, M.R., Matsuuchi, L., Kelly, R.B., and DeFranco, A.L. (1991). Tyrosine phosphorylation of components of the B-cell antigen receptors following receptor crosslinking. Proc Natl Acad Sci U S A 88, 3436-3440.

Goto, T., Kennel, S.J., Abe, M., Takishita, M., Kosaka, M., Solomon, A., and Saito, S. (1994). A novel membrane antigen selectively expressed on terminally differentiated human B cells. Blood $84,1922-1930$.

Griffiths, P.S., Walton, C., Samsell, L., Perez, M.K., and Piedimonte, G. (2016). Maternal highfat hypercaloric diet during pregnancy results in persistent metabolic and respiratory abnormalities in offspring. Pediatr Res 79, 278-286.

Hales, C.N., Barker, D.J., Clark, P.M., Cox, L.J., Fall, C., Osmond, C., and Winter, P.D. (1991). Fetal and infant growth and impaired glucose tolerance at age 64. BMJ 303, 1019-1022.

Harmon, K.A., Gerard, L., Jensen, D.R., Kealey, E.H., Hernandez, T.L., Reece, M.S., Barbour, L.A., and Bessesen, D.H. (2011). Continuous glucose profiles in obese and normal-weight pregnant women on a controlled diet: metabolic determinants of fetal growth. Diabetes Care 34, 2198-2204. 
Kamimae-Lanning, A.N., Krasnow, S.M., Goloviznina, N.A., Zhu, X., Roth-Carter, Q.R., Levasseur, P.R., Jeng, S., McWeeney, S.K., Kurre, P., and Marks, D.L. (2015). Maternal highfat diet and obesity compromise fetal hematopoiesis. Mol Metab 4, 25-38.

Kim, S., Kim, N., Presson, A.P., Metzger, M.E., Bonifacino, A.C., Sehl, M., Chow, S.A., Crooks, G.M., Dunbar, C.E., An, D.S., et al. (2014). Dynamics of HSPC repopulation in nonhuman primates revealed by a decade-long clonal-tracking study. Cell Stem Cell 14, 473-485.

Kowalczyk, M.S., Tirosh, I., Heckl, D., Rao, T.N., Dixit, A., Haas, B.J., Schneider, R.K., Wagers, A.J., Ebert, B.L., and Regev, A. (2015). Single-cell RNA-seq reveals changes in cell cycle and differentiation programs upon aging of hematopoietic stem cells. Genome Res 25, 1860-1872. Krebsbach, P.H., and Villa-Diaz, L.G. (2017). The Role of Integrin alpha6 (CD49f) in Stem Cells: More than a Conserved Biomarker. Stem Cells Dev 26, 1090-1099.

Lee, J.M., Govindarajah, V., Goddard, B., Hinge, A., Muench, D.E., Filippi, M.D., Aronow, B., Cancelas, J.A., Salomonis, N., Grimes, H.L., et al. (2018). Obesity alters the long-term fitness of the hematopoietic stem cell compartment through modulation of Gfi1 expression. J Exp Med $215,627-644$.

Li, M., Sloboda, D.M., and Vickers, M.H. (2011). Maternal obesity and developmental programming of metabolic disorders in offspring: evidence from animal models. Exp Diabetes Res $2011,592408$.

Liu, A., Chen, M., Kumar, R., Stefanovic-Racic, M., O'Doherty, R.M., Ding, Y., Jahnen-Dechent, W., and Borghesi, L. (2018). Bone marrow lympho-myeloid malfunction in obesity requires precursor cell-autonomous TLR4. Nat Commun 9, 708.

Morrison, S.J., Hemmati, H.D., Wandycz, A.M., and Weissman, I.L. (1995). The purification and characterization of fetal liver hematopoietic stem cells. Proc Natl Acad Sci U S A 92, 1030210306. 
Myles, I.A., Fontecilla, N.M., Janelsins, B.M., Vithayathil, P.J., Segre, J.A., and Datta, S.K. (2013). Parental dietary fat intake alters offspring microbiome and immunity. J Immunol 191, 3200-3209.

Nagareddy, P.R., Kraakman, M., Masters, S.L., Stirzaker, R.A., Gorman, D.J., Grant, R.W., Dragoljevic, D., Hong, E.S., Abdel-Latif, A., Smyth, S.S., et al. (2014). Adipose tissue macrophages promote myelopoiesis and monocytosis in obesity. Cell Metab 19, 821-835. Notta, F., Doulatov, S., Laurenti, E., Poeppl, A., Jurisica, I., and Dick, J.E. (2011). Isolation of single human hematopoietic stem cells capable of long-term multilineage engraftment. Science 333, 218-221.

Oben, J.A., Patel, T., Mouralidarane, A., Samuelsson, A.M., Matthews, P., Pombo, J., Morgan, M., McKee, C., Soeda, J., Novelli, M., et al. (2010). Maternal obesity programmes offspring development of non-alcoholic fatty pancreas disease. Biochem Biophys Res Commun 394, 2428.

Odaka, Y., Nakano, M., Tanaka, T., Kaburagi, T., Yoshino, H., Sato-Mito, N., and Sato, K. (2010). The influence of a high-fat dietary environment in the fetal period on postnatal metabolic and immune function. Obesity (Silver Spring) 18, 1688-1694.

Pellin, D., Loperfido, M., Baricordi, C., Wolock, S.L., Montepeloso, A., Weinberg, O.K., Biffi, A., Klein, A.M., and Biasco, L. (2019). A comprehensive single cell transcriptional landscape of human hematopoietic progenitors. Nat Commun 10, 2395.

Qian, H., Tryggvason, K., Jacobsen, S.E., and Ekblom, M. (2006). Contribution of alpha6 integrins to hematopoietic stem and progenitor cell homing to bone marrow and collaboration with alpha4 integrins. Blood 107, 3503-3510.

Radtke, S., Chan, Y.Y., Sippel, T.R., Kiem, H.P., and Rongvaux, A. (2019). MISTRG mice support engraftment and assessment of nonhuman primate hematopoietic stem and progenitor cells. Exp Hematol 70, 31-41 e31. 
Rastogi, S., Rojas, M., Rastogi, D., and Haberman, S. (2015). Neonatal morbidities among fullterm infants born to obese mothers. J Matern Fetal Neonatal Med 28, 829-835.

Robino, J.J., Pamir, N., Rosario, S., Crawford, L.B., Burwitz, B.J., Roberts, C.T., Jr., Kurre, P., and Varlamov, O. (2020). Spatial and biochemical interactions between bone marrow adipose tissue and hematopoietic stem and progenitor cells in rhesus macaques. Bone, 115248.

Shapiro, A.L., Schmiege, S.J., Brinton, J.T., Glueck, D., Crume, T.L., Friedman, J.E., and Dabelea, D. (2015). Testing the fuel-mediated hypothesis: maternal insulin resistance and glucose mediate the association between maternal and neonatal adiposity, the Healthy Start study. Diabetologia 58, 937-941.

Shirasawa, T., Ohnishi, K., Hagiwara, S., Shigemoto, K., Takebe, Y., Rajewsky, K., and Takemori, T. (1993). A novel gene product associated with mu chains in immature B cells. EMBO J 12, 1827-1834.

Soderborg, T.K., Clark, S.E., Mulligan, C.E., Janssen, R.C., Babcock, L., Ir, D., Young, B., Krebs, N., Lemas, D.J., Johnson, L.K., et al. (2018). The gut microbiota in infants of obese mothers increases inflammation and susceptibility to NAFLD. Nat Commun 9, 4462.

Stuart, T., Butler, A., Hoffman, P., Hafemeister, C., Papalexi, E., Mauck, W.M., 3rd, Hao, Y., Stoeckius, M., Smibert, P., and Satija, R. (2019). Comprehensive Integration of Single-Cell Data. Cell 177, 1888-1902 e1821.

Suk, D., Kwak, T., Khawar, N., Vanhorn, S., Salafia, C.M., Gudavalli, M.B., and Narula, P. (2016). Increasing maternal body mass index during pregnancy increases neonatal intensive care unit admission in near and full-term infants. J Matern Fetal Neonatal Med 29, 3249-3253. Sureshchandra, S., Marshall, N.E., and Messaoudi, I. (2019). Impact of pregravid obesity on maternal and fetal immunity: Fertile grounds for reprogramming. J Leukoc Biol 106, 1035-1050. Sureshchandra, S., Wilson, R.M., Rais, M., Marshall, N.E., Purnell, J.Q., Thornburg, K.L., and Messaoudi, I. (2017). Maternal Pregravid Obesity Remodels the DNA Methylation Landscape of Cord Blood Monocytes Disrupting Their Inflammatory Program. J Immunol 199, 2729-2744. 
Sykes, S.M., and Scadden, D.T. (2013). Modeling human hematopoietic stem cell biology in the mouse. Semin Hematol 50, 92-100.

True, C.A., Takahashi, D.L., Burns, S.E., Mishler, E.C., Bond, K.R., Wilcox, M.C., Calhoun, A.R., Bader, L.A., Dean, T.A., Ryan, N.D., et al. (2017). Chronic combined hyperandrogenemia and western-style diet in young female rhesus macaques causes greater metabolic impairments compared to either treatment alone. Hum Reprod 32, 1880-1891.

Varlamov, O., Bishop, C.V., Handu, M., Takahashi, D., Srinivasan, S., White, A., and Roberts, C.T., Jr. (2017). Combined androgen excess and Western-style diet accelerates adipose tissue dysfunction in young adult, female nonhuman primates. Hum Reprod 32, 1892-1902.

Varlamov, O., Bucher, M., Myatt, L., Newman, N., and Grant, K.A. (2020). Daily Ethanol Drinking Followed by an Abstinence Period Impairs Bone Marrow Niche and Mitochondrial Function of Hematopoietic Stem/Progenitor Cells in Rhesus Macaques. Alcohol Clin Exp Res. Wilson, R.M., Marshall, N.E., Jeske, D.R., Purnell, J.Q., Thornburg, K., and Messaoudi, I. (2015). Maternal obesity alters immune cell frequencies and responses in umbilical cord blood samples. Pediatr Allergy Immunol 26, 344-351.

Wilson, R.M., and Messaoudi, I. (2015). The impact of maternal obesity during pregnancy on offspring immunity. Mol Cell Endocrinol 418 Pt 2, 134-142.

Wu, C., Espinoza, D.A., Koelle, S.J., Potter, E.L., Lu, R., Li, B., Yang, D., Fan, X., Donahue, R.E., Roederer, M., et al. (2018). Geographic clonal tracking in macaques provides insights into HSPC migration and differentiation. J Exp Med 215, 217-232.

Zannettino, A.C., Buhring, H.J., Niutta, S., Watt, S.M., Benton, M.A., and Simmons, P.J. (1998).

The sialomucin CD164 (MGC-24v) is an adhesive glycoprotein expressed by human hematopoietic progenitors and bone marrow stromal cells that serves as a potent negative regulator of hematopoiesis. Blood 92, 2613-2628.

Zehentmeier, S., and Pereira, J.P. (2019). Cell circuits and niches controlling B cell development. Immunol Rev 289, 142-157. 


\section{SUPPLEMENTARY FIGURES}

\section{Supplementary Figure 1: Single cell analysis of CD34+ progenitors in the FBM.}

(A) Human HSPCs emerge in the aorta gonad mesonephros during the first trimester (E26-40). FL colonization starts at E28 in humans. During the last trimester, HSPCs migrate from the FL to the FBM. This emergence of HSPCs in the FBM coincides with accelerated fetal growth, the development of the BM vascular system, endochondral ossification, and formation of the central marrow cavity. Hematopoietic development in macaques is unknown. (B) Analysis strategy for scRNA-Seq data. (C) UMAP representation of fetal bone marrow CD34+ cells highlighting differences with maternal diet.

A.
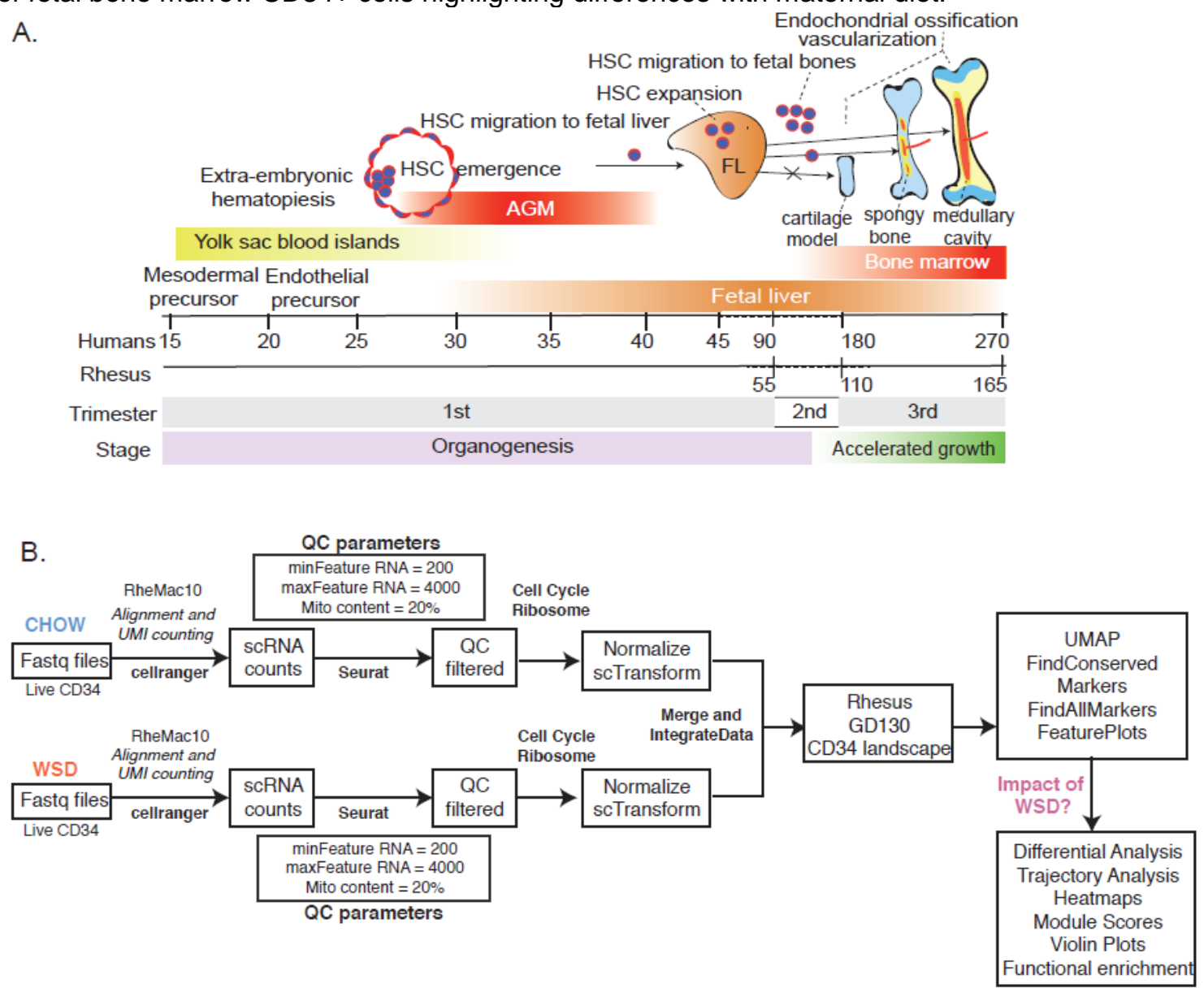

C.

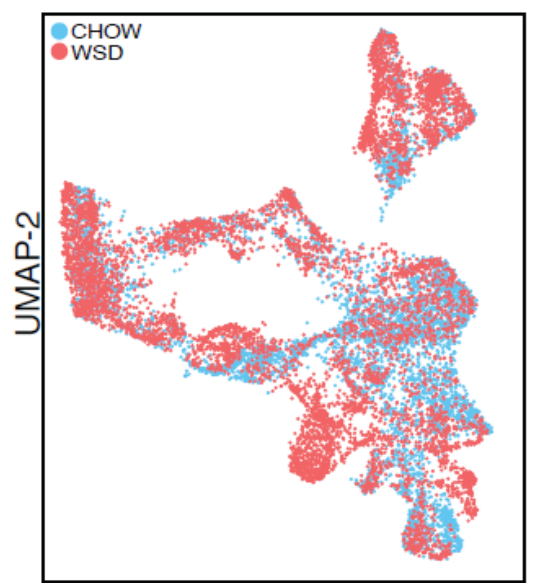

UMAP-1 


\section{Supplementary Figure 2: Phenotypic changes in the FBM lymphoid compartment in response to maternal WSD.}

(A) Uniform Manifold Approximation and Projection (UMAP) of single cell clusters of CD34+ cells $(n=3$ /group, pooled) highlighting the common lymphoid progenitor subsets. (B) Feature plot highlighting markers to delineate CLP-1, CLP-2 and proliferating CLP clusters. (C) Gating strategy for identification of $\mathrm{T}, \mathrm{B}$, and NK-cells in the FBM.
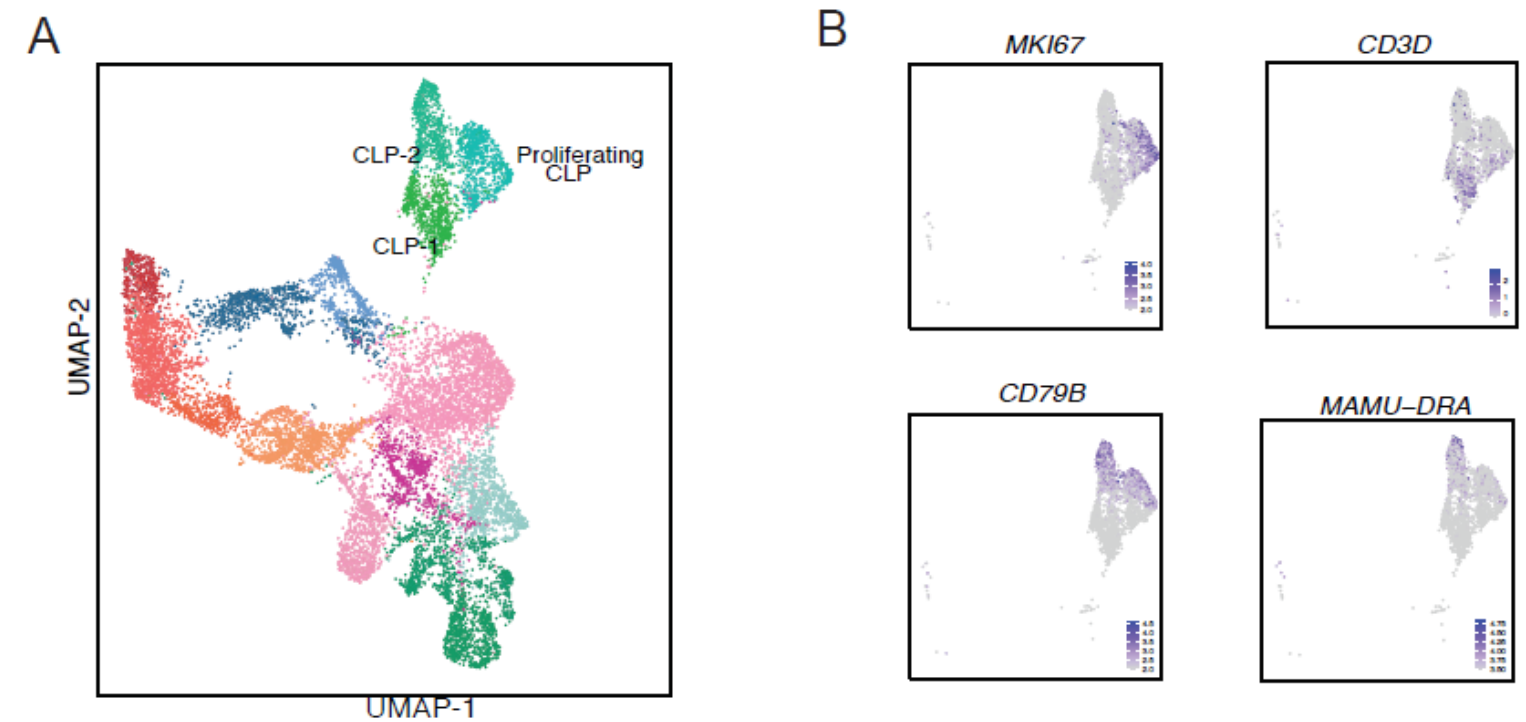

C
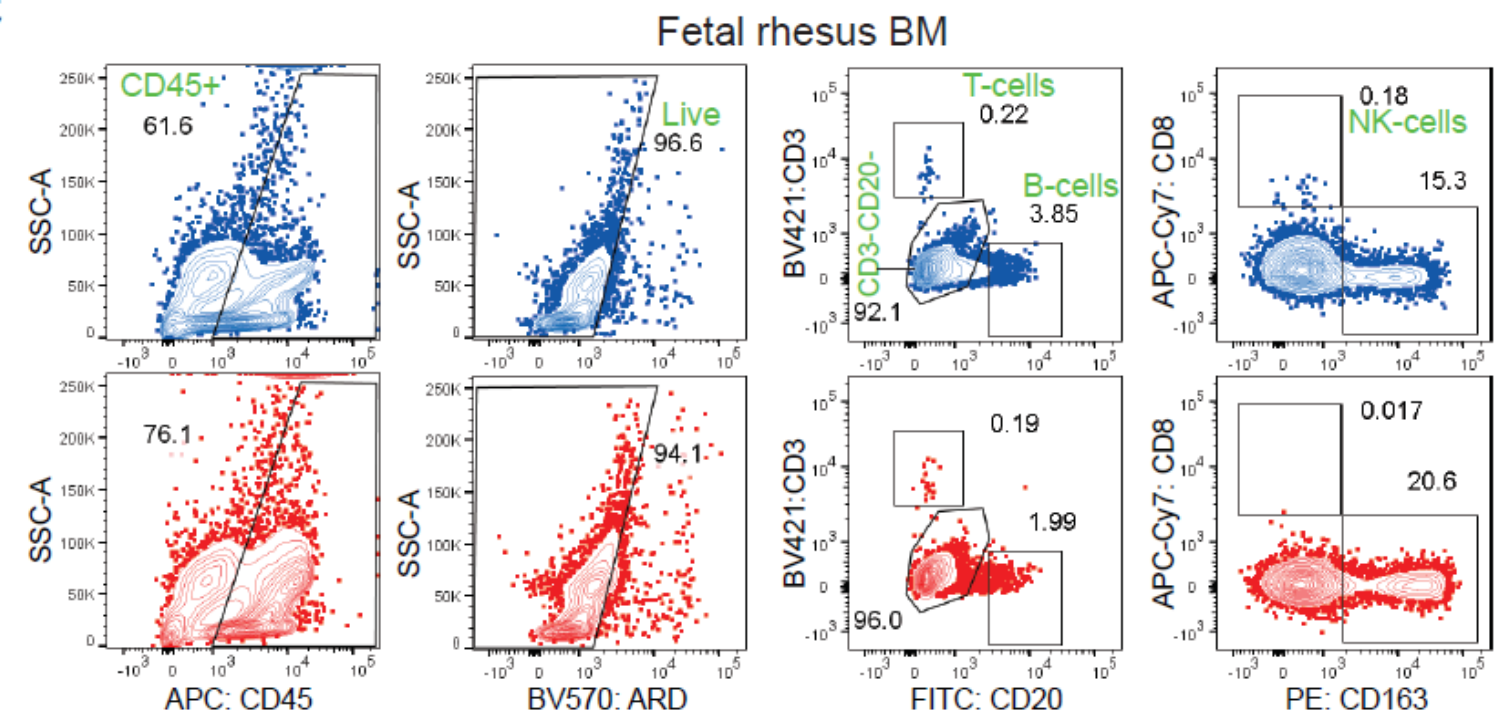

FITC: CD20

PE: CD163 


\section{Supplementary Figure 3: Phenotypic changes in the FBM myeloid compartment in response to maternal WSD.}

(A) Profile of HSCs and myeloid cell progenitors ordered by slingshot pseudotime. (B) Gating strategy to identify bone marrow monocytes and stromal macrophages. (C) Bar graphs comparing relative frequencies of monocytes and macrophages within the FBM mononuclear cell fraction. (D) Gating strategy for identification of TNF $\alpha+$ macrophages/monocytes in response to TLR stimulation. (E) Dot plots comparing TNF $\alpha$ responses following TLR ligand-stimulated FBM monocytes from 4 control and 4 WSD fetuses. Each data point shows the \% of TNF $\alpha+$ cells within the CD11b+HLA-DRhighCD163- gate; horizontal bars are means.

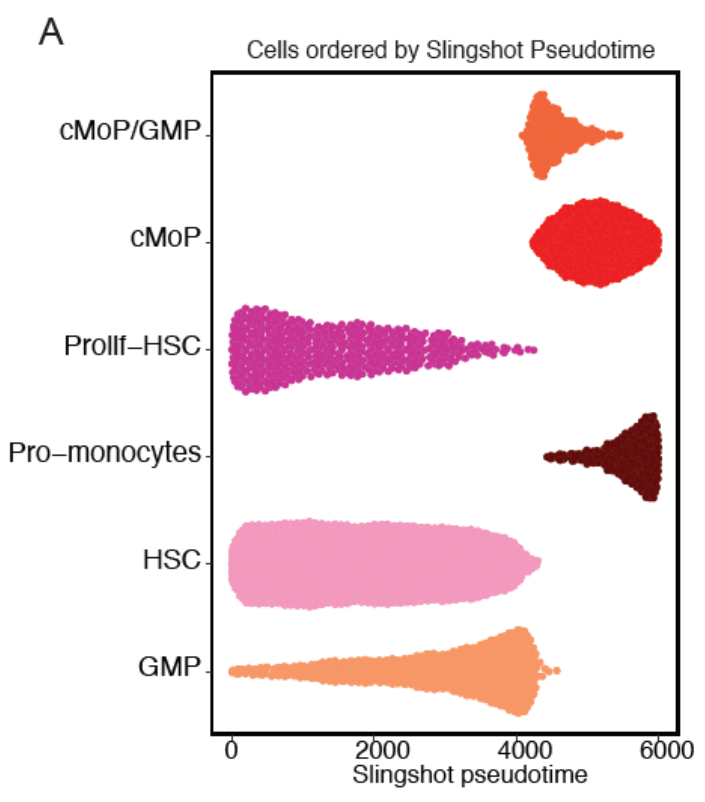

B


C



DRhigh monocytes

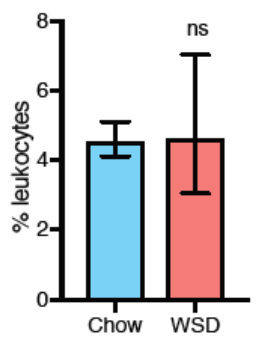

D.
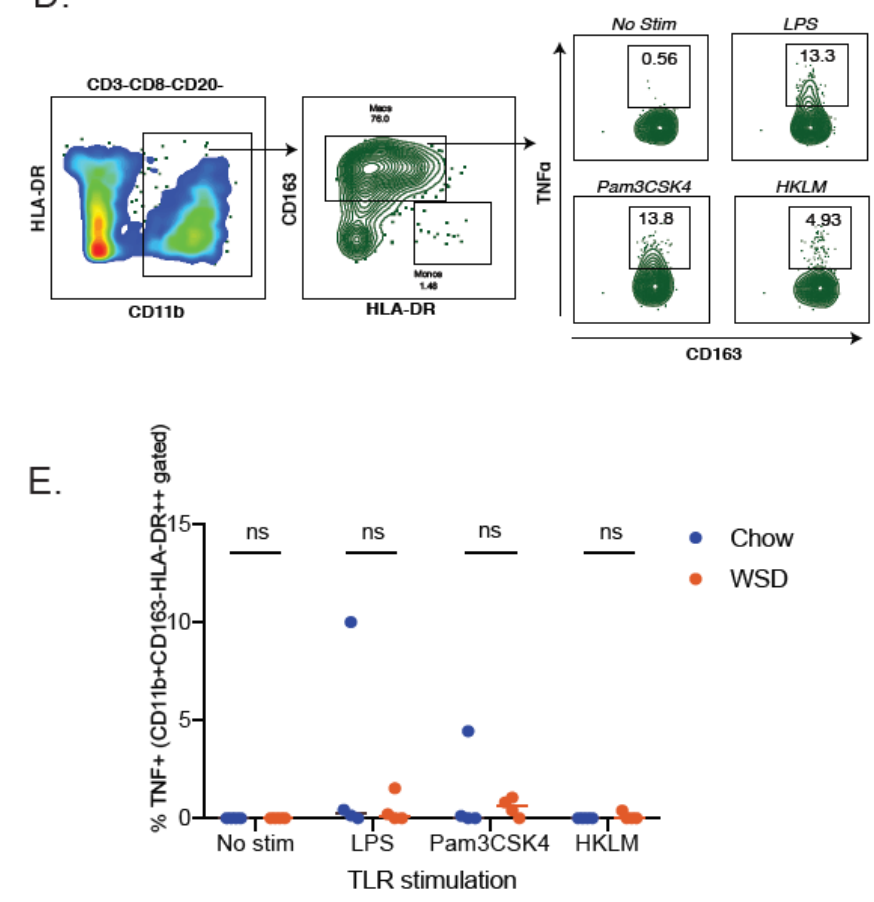
Supplementary Figure 4: Functional impairment of CD34+ HSPCs in response to maternal WSD. (A) Example of flow cytometry analysis of peripheral blood from an NSG mouse engrafted with fetal rhesus CD34+ cells in comparison with unengrafted controls. (B) Relative frequencies of engrafted CD34+ cells. (C) Gating strategy for identification of rhesus HSPCs, T, B, NK-cells in the bone marrow. (D) Relative frequencies of rhesus T, B, and NK-cells in the bone marrow of NSG mice. (E) Gating strategy for identification of monocytes/macrophages and granulocytes from the NSG bone marrow. (F) Gating strategy for identification of recently infiltrating macrophages in the NSG bone marrow/spleen. (G) Gating strategy for identification of rhesus HSPCs, T, B, NK cells in mouse spleen and blood. (H) Relative frequencies of rhesus T, B, and NK-cells in NSG blood. (I) Gating strategy for identification of blood monocyte subsets in NSG mice.

A
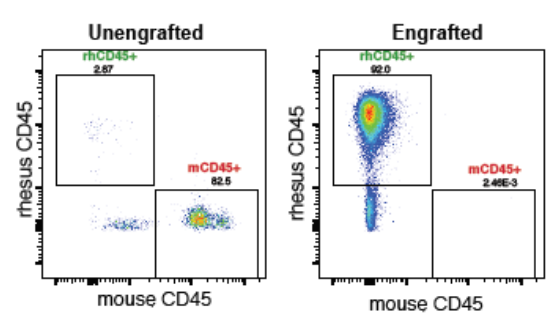

mouse CD45
B

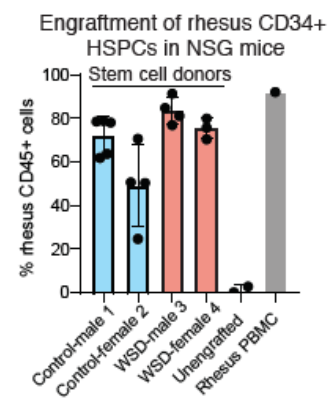

C

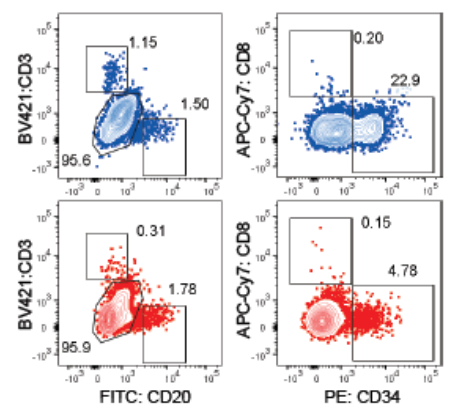

D Bone marrow T cells
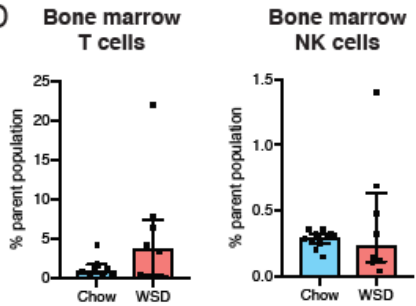
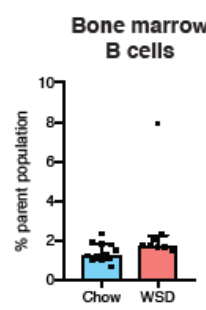

E.

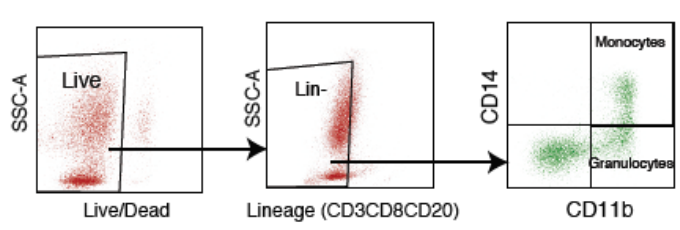

F.

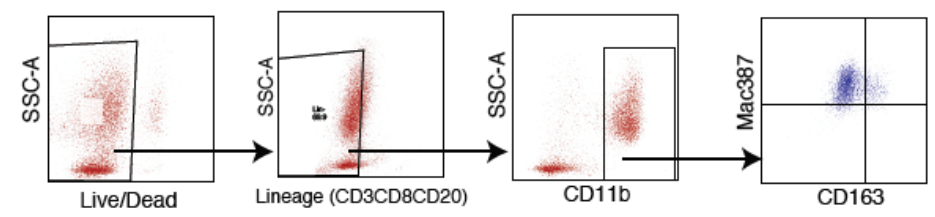

G.



NSG recipient blood

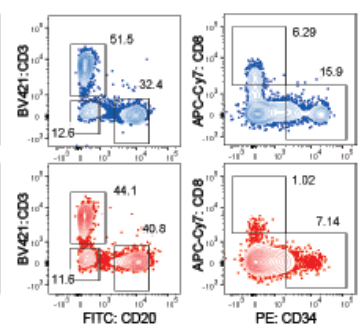

$\mathrm{H}$.
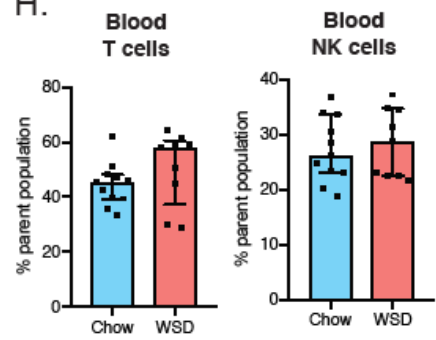

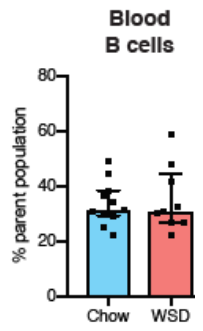

I.
